\title{
THE SIZE OF RANDOM FRAGMENTATION TREES
}

\author{
SVANTE JANSON AND RALPH NEININGER ${ }^{1}$
}

\begin{abstract}
We study a random fragmentation process and its associated random tree. The process has earlier been studied by Dean and Majumdar [7, who found a phase transition: the number of fragmentations is asymptotically normal in some cases but not in others, depending on the position of roots of a certain characteristic equation. This parallels the behaviour of discrete analogues with various random trees that have been studied in computer science. We give rigorous proofs of this phase transition, and add further details.

The proof uses the contraction method. We extend some previous results for recursive sequences of random variables to families of random variables with a continuous parameter; we believe that this extension has independent interest.
\end{abstract}

\section{The PROBlem AND RESUlt}

Consider the following fragmentation process [7. Fix $b \geq 2$ and a random vector $\mathbf{V}=\left(V_{1}, \ldots, V_{b}\right)$. Note that the definitions and results below depend only on the distribution of $\left(V_{1}, \ldots, V_{b}\right)$, so it would be more precise to say that we fix a distribution on $\mathbb{R}^{b}$; we find it, however, more convenient to state the results in terms of a random vector. We assume throughout the paper that $0 \leq V_{j} \leq 1$, $j=1, \ldots, b$, and

$$
\sum_{j=1}^{b} V_{j}=1,
$$

i.e., that $\left(V_{1}, \ldots, V_{b}\right)$ belongs to the standard simplex. For simplicity we also assume that each $V_{j}<1$ a.s. We allow $V_{j}=0$, but note that, a.s., $0<V_{j}<1$ for at least one $j$.

Starting with an object of size $x \geq 1$, we break it into $b$ pieces with sizes $V_{1} x, \ldots, V_{b} x$. Continue recursively with each piece of size $\geq 1$, using new (independent) copies of the random vector $\left(V_{1}, \ldots, V_{b}\right)$ each time. The process terminates a.s. after a finite number of steps, leaving a finite set of fragments of sizes $<1$. We let $N(x)$ be the random number of fragmentation events, i.e., the number of pieces of size $\geq 1$ that appear during the process; further, let $N_{e}(x)$ be the final number of fragments, i.e., the number of pieces of size $<1$ that appear.

Date: September 13, 2006.

${ }^{1}$ Research supported by an Emmy Noether fellowship of the DFG. 
This model has been studied by Dean and Majumdar [7, who found (without giving a rigorous proof) that the asymptotic behaviour of $N(x)$ as $x \rightarrow \infty$ depends on the position of the roots of a certain characteristic equation; we give a precise version of this in Theorem 1.3 below. Some special cases have earlier been studied by other authors, see Section 7

It is natural to consider the fragmentation process as a tree, with the root representing the original object, its children the result of the first fragmentation, and so on. It is then convenient to let the fragmentation go on for ever, although we ignore what happens to pieces smaller than 1 . Let us label each node with the size of the corresponding object.

We thus consider the infinite rooted $b$-ary tree $T_{b}$, whose nodes are the strings $J=j_{1} \cdots j_{k}$ with $j_{i} \in\{1, \ldots, b\}$ and $k \geq 0$. Let $\mathcal{B}^{*}$ denote the set of all such strings, and let $\left(V_{1}^{(J)}, \ldots, V_{b}^{(J)}\right), J \in \mathcal{B}^{*}$, be independent copies of $\mathbf{V}$. Then node $J=j_{1} \cdots j_{k}$ gets the label $x \prod_{i=1}^{k} V_{j_{i}}^{\left(j_{1} \cdots j_{i-1}\right)}$. Thus $N(x)$ is the number of nodes with labels $\geq 1$, i.e.

$$
N(x)=\sum_{J \in \mathcal{B}^{*}} \mathbf{1}_{\left\{x \prod_{i=1}^{k} V_{j_{i}}^{\left(j_{1} \cdots j_{i-1}\right)} \geq 1\right\}}
$$

By the recursive construction of the fragmentation process, we have $N(x)=0$ for $0 \leq x<1$ and

$$
N(x) \stackrel{\mathrm{d}}{=} 1+\sum_{j=1}^{b} N^{(j)}\left(V_{j} x\right), \quad x \geq 1,
$$

where $N^{(j)}(\cdot)$ are copies of the process $N(\cdot)$, independent of each other and of $\left(V_{1}, \ldots, V_{b}\right)$.

Remark 1.1. Let $\mathcal{T}(x)$ be the subtree of $\mathcal{T}(\infty)=T_{b}$ consisting of all nodes with labels $\geq 1$. Then $N(x)=|\mathcal{T}(x)|$, the number of nodes in $\mathcal{T}(x)$. More precisely, we call these nodes internal nodes of $\mathcal{T}(x)$, and we say that a node in $\mathcal{T}(\infty)$ is an external node of $\mathcal{T}(x)$ if it has label $<1$ but its parent is an internal node.

Thus $N(x)$ is the number of internal nodes, and $N_{e}(x)$ is the number of external nodes. Since each internal node has $b$ internal or external children, we have, for $x \geq 1, N(x)+N_{e}(x)=1+b N(x)$, or $N_{e}(x)=(b-1) N(x)+1$. Hence the results for $N(x)$ immediately yield similar results for $N_{e}(x)$ and the total number of external and internal nodes $N(x)+N_{e}(x)$ too.

In this paper we thus study the size of the fragmentation tree $\mathcal{T}(x)$. Of course, it is interesting to study other properties too, such as height, pathlength, ....

Note that we may define $\mathcal{T}(x)$ for all $x \geq 0$ simultaneously, using the same $V_{j}^{(J)}$; this defines $(\mathcal{T}(x))_{x \geq 0}$ as an increasing stochastic process of trees. (Equivalently, we can label all nodes of $\mathcal{T}(\infty)$ as above, starting with 1 at the root, and then keep all nodes with labels $\geq 1 / x)$. 
Remark 1.2. We assume for convenience that each object is split into the same number $b$ of parts. Our method applies also to the case of a random number of parts. Indeed, if the number of parts is bounded, we can use the results below with $b$ large enough, setting the non-existing $V_{j}:=0$. If the number of parts is unbounded, we can, under suitable assumptions, use the proofs below with minor modifications. We leave this extension to the reader.

Our main result is Theorem 1.3 below on the asymptotic distribution of $N(x)$, together with the corresponding estimates for mean and variance given in Theorem 3.1

We define (with $0^{z}:=0$ ), at least for $\operatorname{Re} z \geq 0$,

$$
\phi(z):=\sum_{j=1}^{b} \mathbb{E} V_{j}^{z},
$$

and note that $\phi(z)$ is bounded and analytic in the open right half-plane $\{z: \operatorname{Re} z>$ $0\}$. Since we assume (1.1), clearly $\phi(1)=1$. Since further $0 \leq V_{j}<1$ a.s., the function $\phi(z)$ is decreasing for real $z>0$; hence $\phi(z)>1$ when $0<z<1$ and $\phi(z)<1$ for $1<z<\infty$. Further, $|\phi(z)| \leq \sum_{j} \mathbb{E}\left|V_{j}^{z}\right|=\phi(\operatorname{Re} z)$, so $|\phi(z)|<1$ when $\operatorname{Re} z>1$.

A crucial role is played by the solutions to the characteristic equation

$$
\phi(\lambda)=1
$$

By the comments above, $\lambda=1$ is one root, and $\operatorname{Re} \lambda \leq 1$ for every root $\lambda$; furthermore, there is no real root in $(0,1)$.

We further define

$$
\alpha:=-\phi^{\prime}(1)=\sum_{j=1}^{b} \mathbb{E}\left(-V_{j} \ln V_{j}\right),
$$

the expected entropy of $\left(V_{1}, \ldots, V_{b}\right)$.

We need a (weak) regularity condition on the distribution of $\left(V_{1}, \ldots, V_{b}\right)$. We find the following convenient, although it can be weakened to Condition $\mathrm{B}(\delta)$ in Section 2 for suitable $\delta$. For examples where this regularity and Theorem 1.3 fail, see Example 8.1

Condition A. Each $V_{j}$ has a distribution that is absolutely continuous on $(0,1)$, although a point mass at 0 is allowed.

Note that there is no condition on the joint distribution. In one case, however, we need a condition including the joint distribution too. (Note that both conditions are satisfied if $\mathbf{V}$ has a density on the standard simplex, i.e. if $\left(V_{1}, \ldots, V_{b-1}\right)$ has a density.) 
Condition $\mathbf{A}^{\prime}$. The support of the distribution of $\mathbf{V}$ on the standard simplex has an interior point.

If Condition A holds, then, by Lemmas 2.2 and 2.1 below, there is only a finite number of roots of $\phi(\lambda)=1$ in $\{\lambda: \operatorname{Re} \lambda \geq \delta\}$ for any $\delta>0$. We may thus order the roots with $\operatorname{Re} \lambda>0$ as $\lambda_{1}, \lambda_{2}, \ldots, \lambda_{M}$ with decreasing real parts: $\lambda_{1}=1>$ $\operatorname{Re} \lambda_{2} \geq \operatorname{Re} \lambda_{3} \geq \ldots$; we will assume this in the sequel. If $\lambda_{1}=1$ is the only root with $\operatorname{Re} \lambda>0$, we set $\lambda_{2}=-\infty$ for convenience.

We let $\mathcal{M}^{\mathbb{C}}$ denote the space of probability measures on $\mathbb{C}$, and let

$$
\mathcal{M}_{2}^{\mathbb{C}}(\gamma):=\left\{\eta \in \mathcal{M}^{\mathbb{C}}: \int|z|^{2} d \eta(z)<\infty, \text { and } \int z d \eta(z)=\gamma\right\}, \quad \gamma \in \mathbb{C} .
$$

We let $T$ denote the map (assuming $\lambda_{2} \neq-\infty$ )

$$
T: \mathcal{M}^{\mathbb{C}} \rightarrow \mathcal{M}^{\mathbb{C}}, \quad \eta \mapsto \mathcal{L}\left(\sum_{r=1}^{b} V_{r}^{\lambda_{2}} Z^{(r)}\right),
$$

where $\left(V_{1}, \ldots, V_{b}\right), Z^{(1)}, \ldots, Z^{(b)}$ are independent and $\mathcal{L}\left(Z^{(r)}\right)=\eta$ for $r=1, \ldots, b$. Note that $T$ maps $\mathcal{M}_{2}^{\mathbb{C}}(\gamma)$ into itself for each $\gamma$, since $\lambda_{2}$ satisfies $\phi\left(\lambda_{2}\right)=1$.

We state our main result. The constant $\alpha>0$ is defined in (1.6) above and $\beta$ is given explicitly in Theorem 3.1 The $\ell_{2}$ distance between distributions is defined in Section 4 .

Theorem 1.3. Suppose that Condition A holds. Then we have:

(i) If $\operatorname{Re} \lambda_{2}<1 / 2$ then $\mathbb{E} N(x)=\alpha^{-1} x+o(\sqrt{x})$, Var $N(x) \sim \beta x$ with $\beta>0$ and

$$
\frac{N(x)-\alpha^{-1} x}{\sqrt{x}} \stackrel{\mathrm{d}}{\rightarrow} \mathcal{N}(0, \beta) .
$$

(ii) If $\operatorname{Re} \lambda_{2}=1 / 2$ and each root $\lambda_{i}$ with $\operatorname{Re} \lambda_{i}=1 / 2$ is a simple root of $\phi(\lambda)=1$, and further Condition $\mathrm{A}^{\prime}$ too holds, then $\mathbb{E} N(x)=\alpha^{-1} x+O(\sqrt{x})$, $\operatorname{Var}(N(x)) \sim \beta x \ln x$ with $\beta>0$ and

$$
\frac{N(x)-\alpha^{-1} x}{\sqrt{x \ln x}} \stackrel{\mathrm{d}}{\rightarrow} \mathcal{N}(0, \beta) .
$$

(iii) If $\operatorname{Re} \lambda_{2}>1 / 2$, and $\lambda_{2}$ and $\lambda_{3}=\overline{\lambda_{2}}$ are the only roots of (1.5) with this real part, and these roots are simple, then $\mathbb{E} N(x)=\alpha^{-1} x+\operatorname{Re}\left(\gamma x^{\lambda_{2}}\right)+O\left(x^{\kappa}\right)$, with $\alpha^{-1}>0, \gamma \in \mathbb{C} \backslash\{0\}, 1 / 2<\kappa<\operatorname{Re} \lambda_{2}$ and

$$
\ell_{2}\left(\frac{N(x)-\alpha^{-1} x}{x^{\operatorname{Re} \lambda_{2}}}, \operatorname{Re}\left(\Xi e^{\mathrm{i} \operatorname{Im} \lambda_{2} \ln x}\right)\right)=O\left(x^{\kappa-\operatorname{Re} \lambda_{2}}\right),
$$

for some complex random variable $\Xi$. Furthermore, $\mathcal{L}(\Xi)$ is the unique fixed point of $T$ in $\mathcal{M}_{2}^{\mathbb{C}}(\gamma)$. 
Remark 1.4. We can regard our process as a general (age-dependent) branching process [16. Chapter 6], provided we make a logarithmic change of time as in Section 3 Indeed, there are two versions. For internal nodes, the individuals in the branching process live for ever, and give birth at times $-\ln V_{1}, \ldots,-\ln V_{d}$. For external nodes, we have a splitting process where each individual when it dies gives birth to new particles with life lengths $-\ln V_{1}, \ldots,-\ln V_{d}$. For both versions, we obtain a super-critical branching process with Malthusian parameter 1, but the identity (1.1) causes the asymptotics for moments and distributions to be quite different from typical super-critical branching processes.

Remark 1.5. If $\lambda_{2}$ in (1.7) is real, then the stable distributions of index $1 / \lambda_{2}$ are fixed points of $T$. Note, however, that in our case, $\lambda_{2}$ never is real. Moreover, the fixed points we are interested in have finite variance, and are thus quite different from stable distributions.

For the related Quicksort fixed point equation, Fill and Janson [10] found a complete characterization of the set of fixed points; in that case, all fixed points are formed by combining certain stable distributions with the unique fixed point with mean 0 and finite variance.

Remark 1.6. Condition $\mathrm{A}^{\prime}$ is needed only in part (ii), and is needed only to exclude the possibility that for each root $\lambda_{i}$ with $\operatorname{Re} \lambda_{i}=1 / 2$, there is a complex constant $C_{i}$ such that

$$
\sum_{j=1}^{b} V_{j}^{\lambda_{i}}=C_{i}
$$

This is easily seen to be impossible if Condition $\mathrm{A}^{\prime}$ holds, and even otherwise it seems highly unlikely for any particular example, but it seems very likely that there are examples satisfying Condition A where $\mathbf{V}$ is concentrated on a curve, say, such that (1.8) holds.

We will prove the statements on mean and variance, with further refinements, in Section 3. To prove convergence in distribution, we will use a continuous time version of the contraction method. We develop a general theorem, that we find to be of independent interest, in Section 5. This theorem is applied to our problem in Section 6] Some examples are given in Sections 7 and 8 .

Acknowledgements. This research was initiated and largely done during conferences in Oberwolfach, Frankfurt and Vienna in August and September 2004. We thank Luc Devroye, Jim Fill, Allan Gut and Hsien-Kuei Hwang for valuable comments. 


\section{Further PRELIMINARIES}

We define (again with $0^{z}:=0$ )

$$
\psi(z, w):=\operatorname{Cov}\left(\sum_{j=1}^{b} V_{j}^{z}, \sum_{j=1}^{b} V_{j}^{w}\right)=\mathbb{E}\left(\sum_{j=1}^{b} V_{j}^{z} \sum_{k=1}^{b} V_{k}^{w}\right)-\phi(z) \phi(w) .
$$

In particular, $\psi(z, \bar{z})=\mathbb{E}\left|\sum_{j=1}^{b} V_{j}^{z}-\phi(z)\right|^{2} \geq 0$, with equality only if $\sum_{j=1}^{b} V_{j}^{z}=$ $\phi(z)$ a.s.

For $\operatorname{Re} z, \operatorname{Re} w \geq 0$, we have $\left|V_{j}^{z}\right|,\left|V_{j}^{w}\right| \leq 1$ and thus $|\psi(z, w)| \leq 2 b^{2}$.

We say that $\mathbf{V}$ is lattice if there exists a number $r$ with $0<r<1$ such that every $V_{j} \in\left\{r^{n}\right\}_{n \geq 0} \cup\{0\}$ a.s.; otherwise $\mathbf{V}$ is non-lattice. Basic Fourier analysis applied to the probability measure $\nu$ defined in (3.16) shows that $\mathbf{V}$ is non-lattice if and only if $\lambda=1$ is the only root of (1.5) with $\operatorname{Re} \lambda=1$. (Otherwise, there is an infinite number of roots with $\operatorname{Re} \lambda=1$.) We will assume this, and more, below.

We introduce a family of regularity conditions that are weaker than Condition A.

Condition $\mathbf{B}(\delta)$. (Here $\delta$ is a real number with $\delta \geq 0$.)

$$
\limsup _{t \rightarrow \infty}|\phi(\delta+\mathrm{i} t)|<1 .
$$

Lemma 2.1. If Condition $\mathrm{B}(\delta)$ holds for some $\delta \geq 0$, then Condition $\mathrm{B}\left(\delta^{\prime}\right)$ holds for every $\delta^{\prime}>\delta$ as well; moreover

$$
\limsup _{\substack{\operatorname{Re} \\ \operatorname{Im} z \rightarrow \infty}}|\phi(z)|<1 .
$$

Proof. Choose first $\varepsilon>0$ such that $\limsup _{t \rightarrow \infty}|\phi(\delta+\mathrm{i} t)|<1-2 \varepsilon$, and then $A$ such that $|\phi(\delta+\mathrm{i} t)| \leq 1-2 \varepsilon$ if $t \geq A$, and thus also if $t \leq-A$. Recall further that $|\phi(\delta+\mathrm{i} t)| \leq b$ for all $t$. Since $\phi(z)$ is analytic, and thus harmonic, in the half-plane $\mathcal{H}_{\delta}:=\{z: \operatorname{Re} z>\delta\}$ and bounded and continuous in $\overline{\mathcal{H}_{\delta}}, \phi$ is given by the Poisson integral of its boundary values [13, Lemma 3.4]:

$$
\phi(x+\mathrm{i} y)=\int_{-\infty}^{\infty} P_{x-\delta}(y-t) \phi(\delta+\mathrm{i} t) d t, \quad x>\delta
$$

where $P_{x}(y)=x /\left(\pi\left(x^{2}+y^{2}\right)\right)$, the Poisson kernel for the right half-plane. Let $\omega(x+\mathrm{i} y):=\int_{-A}^{A} P_{x-\delta}(y-t) d t$, the harmonic measure of $[\delta-\mathrm{i} A, \delta+\mathrm{i} A]$; then (2.2) implies

$$
|\phi(x+\mathrm{i} y)| \leq \int_{-\infty}^{\infty} P_{x-\delta}(y-t)|\phi(\delta+\mathrm{i} t)| d t \leq b \omega(x+\mathrm{i} y)+1-2 \varepsilon .
$$

It is well-known, and easy to see, that the set $B:=\left\{z \in \mathcal{H}_{\delta}: \omega(z)>\varepsilon / b\right\}$ is bounded; in fact, it is the intersection of $\mathcal{H}_{\delta}$ and a circular disc [13, p. 13]. Thus, $A_{1}:=\sup \{\operatorname{Im} z: z \in B\}<\infty$, and if $\operatorname{Re} x \geq \delta$ and $|y|>A_{1}$, then $\omega(z) \leq \varepsilon / b$ and (2.3) yields $|\phi(x+\mathrm{i} y)| \leq 1-\varepsilon$. 
Lemma 2.2. If Condition $\mathrm{A}$ holds, then Condition $\mathrm{B}(\delta)$ holds for every $\delta \geq 0$.

Proof. We have $\mathbb{E} V_{j}^{\mathrm{it}}=\mathbb{E}\left(e^{\mathrm{i} t \ln V_{j}} \mathbf{1}_{\left\{V_{j}>0\right\}}\right)$, the Fourier transform of the distribution of $\ln V_{j}$ (ignoring any point mass at 0 ), so by Condition $\mathrm{A}$ and the RiemannLebesgue lemma, $\mathbb{E} V_{j}^{\text {it }} \rightarrow 0$ as $t \rightarrow \infty$ for every $j$, and thus $\phi(i t) \rightarrow 0$ as $t \rightarrow \infty$. Hence, Condition B(0) holds, and the result follows by Lemma 2.1

Lemma 2.3. If Condition $\mathrm{B}(\delta)$ holds for some $\delta>0$, then there is only a finite number of roots to $\phi(\lambda)=1$ with $\operatorname{Re} \lambda \geq \delta$.

Proof. By Lemma 2.1] all such roots satisfy $|\operatorname{Im} \lambda| \leq C$ for some $C<\infty$. Furthermore, all roots satisfy $\operatorname{Re} \lambda \leq 1$, so if further $\operatorname{Re} \lambda \geq \delta, \lambda$ belongs to a compact rectangle $K$ in the open right half-plane. Since, $\phi(z)-1$ is analytic and non-constant in this half-plane, it has only a finite number of roots in $K$.

In particular, by the comments above, Condition $\mathrm{B}(\delta)$ with $\delta \leq 1$ implies that $\mathbf{V}$ is non-lattice.

\section{MEAN AND VARIANCE}

We let $\Lambda$ denote the set of solutions to the characteristic equation (1.5), i.e.

$$
\Lambda:=\{\lambda: \phi(\lambda)=1\}
$$

we further define its subsets

$$
\Lambda(s):=\{z \in \Lambda: \operatorname{Re}(z)=s\} .
$$

In general, $\phi(\lambda)$ is defined only for $\operatorname{Re} \lambda \geq 0$, and we consider only such $\lambda$ in (3.1). However, in cases where $\phi$ extends to a meromorphic function in a larger domain (for example, when $\phi$ is rational), we may include such $\lambda$ too in $\Lambda$; this makes no difference in Theorem 3.1. (In Theorem 3.4, we include all roots in the complex plane.) We will use $\Lambda(s)$ only for $s \geq 0$, where there is no ambiguity.

Let $m(x):=\mathbb{E} N(x)$ and $\sigma^{2}(x):=\operatorname{Var} N(x)$. We will show the following asymptotics.

Theorem 3.1. Assume that Condition $\mathrm{B}(\delta)$ holds with $0 \leq \delta<1$, and let $\lambda_{1}, \ldots, \lambda_{M}$ be the elements of $\{\lambda \in \Lambda: \operatorname{Re} \lambda>\delta\}$, ordered with $\lambda_{1}=1>\operatorname{Re} \lambda_{2} \geq$ $\operatorname{Re} \lambda_{3} \geq \cdots$. Then, the following hold as $x \rightarrow \infty$ :

(i) $m(x) \sim \alpha^{-1} x$.

(ii) If further $\phi^{\prime}\left(\lambda_{i}\right) \neq 0$ for $i=1, \ldots, M$, i.e., each $\lambda_{i}$ is a simple root of $\phi(\lambda)=1$, then, more precisely, for every $\delta^{\prime}>\delta$,

$$
m(x)=\sum_{i=1}^{M} \frac{1}{-\lambda_{i} \phi^{\prime}\left(\lambda_{i}\right)} x^{\lambda_{i}}+O\left(x^{\delta^{\prime}}\right) .
$$


(iii) If $\delta<1 / 2$ and either $M=1$ or $\operatorname{Re} \lambda_{2}<1 / 2$, then $\sigma^{2}(x) \sim \beta x$, with

$$
\beta=\alpha^{-1} \frac{1}{2 \pi} \int_{-\infty}^{\infty} \frac{\psi(1 / 2+\mathrm{i} u, 1 / 2-\mathrm{i} u)}{|1 / 2+\mathrm{i} u|^{2}|1-\phi(1 / 2+\mathrm{i} u)|^{2}} d u \in(0, \infty)
$$

(iv) If $M \geq 2$ and $\operatorname{Re} \lambda_{2}=1 / 2$, and each $\lambda_{i}$ with $\operatorname{Re} \lambda_{i}=1 / 2$ is a simple root of $\phi(\lambda)=1$, then $\sigma^{2}(x)=\beta x \ln x+o(x \ln x)$, with

$$
\beta=\sum_{\lambda \in \Lambda(1 / 2)} \frac{1}{\alpha\left|\lambda \phi^{\prime}(\lambda)\right|^{2}} \psi(\lambda, \bar{\lambda}) \geq 0
$$

If, moreover, Condition $\mathrm{A}^{\prime}$ holds (or, more generally, for some $\lambda_{i} \in \Lambda(1 / 2)$, (1.8) does not hold), then $\beta>0$.

(v) If $M \geq 2$ and $\operatorname{Re} \lambda_{2}>1 / 2$, and each $\lambda_{i}$ with $\operatorname{Re} \lambda_{i}=\operatorname{Re} \lambda_{2}$ is a simple root of $\phi(\lambda)=1$, then

$$
\sigma^{2}(x)=\sum_{\lambda_{i}, \lambda_{k} \in \Lambda\left(\operatorname{Re} \lambda_{2}\right)} \frac{1}{\lambda_{i} \lambda_{k} \phi^{\prime}\left(\lambda_{i}\right) \phi^{\prime}\left(\lambda_{k}\right)\left(1-\phi\left(\lambda_{i}+\lambda_{k}\right)\right)} \psi\left(\lambda_{i}, \lambda_{k}\right) x^{\lambda_{i}+\lambda_{k}}+o\left(x^{2 \operatorname{Re} \lambda_{2}}\right) .
$$

Remark 3.2. It follows from the proof that for (i) we do not need Condition $\mathrm{B}(\delta)$; it is enough that $\mathbf{V}$ is non-lattice.

Remark 3.3. The case when some $\phi^{\prime}\left(\lambda_{i}\right)=0$ is similar; now terms $x^{\lambda_{i}} \ln x$ (and possibly $x^{\lambda_{i}} \ln ^{d} x, d \geq 2$ ) will appear in (3.3). We leave the details to the reader.

If $\phi$ is a rational function, then (3.3) can be improved to an exact formula. Furthermore, in case (iii) of Theorem 3.1 we then can give an alternative formula for $\beta$.

Theorem 3.4. Assume that $\phi$ is (i.e., extends to) a rational fumction, and let $\lambda_{1}, \ldots, \lambda_{M}$ be the roots of $\phi(\lambda)=1$ in the complex plane. Suppose further that all these roots are simple.

(i) Then

$$
m(x)=\sum_{i=1}^{M} \frac{1}{-\lambda_{i} \phi^{\prime}\left(\lambda_{i}\right)} x^{\lambda_{i}}-\frac{1}{\phi(0)-1}, \quad x \geq 1 .
$$

(ii) Assume further that $\lambda_{1}=1$ and $\operatorname{Re} \lambda_{i}<1 / 2$ for $i=2, \ldots, M$, and that $V_{j}>0$ a.s. for every $j$. Define, for notational convenience, $\lambda_{0}:=0, a_{0}:=$ $-1 /(b-1)$ and $a_{i}:=-1 /\left(\lambda_{i} \phi^{\prime}\left(\lambda_{i}\right)\right)$ for $i=1, \ldots, M$. Then $\sigma^{2}(x) \sim \beta x$, 
with

$$
\begin{aligned}
\beta=\alpha^{-1} & \sum_{i, k \neq 1} \frac{a_{i} a_{k}}{1-\lambda_{i}-\lambda_{k}}\left(\sum_{j, l=1}^{b} \mathbb{E} V_{j}^{\lambda_{i}} V_{l}^{\lambda_{k}}\left(V_{j} \wedge V_{l}\right)^{1-\lambda_{i}-\lambda_{k}}-2 \phi\left(1-\lambda_{k}\right)+1\right) \\
& -2 \alpha^{-2} \sum_{i=2}^{M} \frac{a_{i}}{\lambda_{i}}\left(\sum_{j, l=1}^{b} \mathbb{E}\left(V_{j}^{\lambda_{i}} V_{l}^{1-\lambda_{i}}-V_{l}\right) \mathbf{1}_{\left\{V_{l} \leq V_{j}\right\}}-\phi\left(1-\lambda_{i}\right)+1\right) \\
& -2 \alpha^{-2} a_{0}\left(\sum_{j, l=1}^{b} \mathbb{E} V_{l}\left(\ln V_{j}-\ln V_{l}\right) \mathbf{1}_{\left\{V_{l}<V_{j}\right\}}-\alpha\right) \\
& +\alpha^{-3}\left(\sum_{j, l=1}^{b} \mathbb{E}\left(V_{j} \wedge V_{l}\right)-1\right)-\alpha^{-1} .
\end{aligned}
$$

The proof of these theorems will occupy the remainder of this section. We first show that all moments of $N(x)$ are finite.

Lemma 3.5. For every $m \geq 1$ and $x \geq 0$, $\mathbb{E} N(x)^{m}<\infty$. Furthermore, $\sup _{0 \leq y \leq x} \mathbb{E} N(y)^{m}<\infty$.

Proof. For a string $J=j_{1} \cdots j_{k} \in \mathcal{B}^{*}$ we denote by $|J|=k$ the depth of the corresponding node in $T_{b}$. Note that we have $\left|\left\{J \in \mathcal{B}^{*}: 0 \leq|J| \leq k\right\}\right| \leq b^{k+1}$. Hence, if $N(x)>b^{k+1}$ for some $k \geq 0$ then by (1.2) there exists a $J=j_{1} \cdots j_{k} \in \mathcal{B}^{*}$ with $x \prod_{i=1}^{k} V_{j_{i}}^{\left(j_{1} \cdots j_{i-1}\right)} \geq 1$. Markov's inequality implies that for all $q \geq 1$

$$
\begin{aligned}
\mathbb{P}\left(N(x)>b^{k+1}\right) & \leq \mathbb{P}\left(\bigcup_{J \in \mathcal{B}^{*}:|J|=k}\left\{\prod_{i=1}^{k} V_{j_{i}}^{\left(j_{1} \cdots j_{i-1}\right)} \geq 1 / x\right\}\right) \\
& \leq \sum_{J \in \mathcal{B}^{*}:|J|=k} \mathbb{P}\left(\prod_{i=1}^{k} V_{j_{i}}^{\left(j_{1} \cdots j_{i-1}\right)} \geq 1 / x\right) \\
& \leq \sum_{J \in \mathcal{B}^{*}:|J|=k} x^{q} \mathbb{E} \prod_{i=1}^{k}\left(V_{j_{i}}^{\left(j_{1} \cdots j_{i-1}\right)}\right)^{q} \\
& =x^{q} \phi(q)^{k} .
\end{aligned}
$$

Hence, for all $y \geq b$ we obtain with $k=\left\lfloor\log _{b} y\right\rfloor-1$ and $\phi(q) \leq 1$ that

$$
\mathbb{P}(N(x)>y) \leq x^{q} \phi(q)^{k} \leq x^{q} \phi(q)^{\log _{b} y-2}=\frac{x^{q}}{\phi(q)^{2}} y^{\log _{b} \phi(q)} .
$$

We have $\phi(q) \rightarrow 0$ as $q \rightarrow \infty$ since $V_{j}<1$ a.s. and by dominated convergence. Hence, for all $m \geq 1$ there exists a $q>0$ with $\log _{b} \phi(q)<-m$. The tail bound (3.7) thus implies $\mathbb{E} N(x)^{m}<\infty$ for all $m \geq 1$ and all $x \geq 0$.

The final statement follows because $0 \leq N(y) \leq N(x)$ when $0 \leq y \leq x$. 
We find it convenient to switch from multiplicative to additive notion. We therefore define

$$
\begin{aligned}
X_{j} & :=-\ln V_{j} \in(0, \infty], \quad j=1, \ldots, b, \\
N_{*}(t) & :=N\left(e^{t}\right), \quad-\infty \leq t<\infty .
\end{aligned}
$$

The definition (1.2) and the recursive equation (1.3) thus translate to

$$
\begin{aligned}
& N_{*}(t)=\sum_{J \in \mathcal{B}^{*}} \mathbf{1}_{\left\{\sum_{i=1}^{k} X_{j_{i}}^{\left(j_{1} \cdots j_{i-1}\right)} \leq t\right\}}, \\
& N_{*}(t) \stackrel{\mathrm{d}}{=} 1+\sum_{j=1}^{b} N_{*}^{(j)}\left(t-X_{j}\right), \quad t \geq 0,
\end{aligned}
$$

where $N_{*}^{(j)}(\cdot)$ are independent copies of the process $N_{*}(\cdot)$, and $N_{*}(t)=0$ for $-\infty \leq$ $t<0$. Further define

$$
\begin{aligned}
m_{*}(t) & :=\mathbb{E} N_{*}(t)=m\left(e^{t}\right), \\
\sigma_{*}^{2}(t) & :=\operatorname{Var} N_{*}(t)=\sigma^{2}\left(e^{t}\right) .
\end{aligned}
$$

Thus $m_{*}(t)=\sigma_{*}^{2}(t)=0$ for $t<0$. Taking expectations in (3.9) we find

$$
m_{*}(t)=1+\mathbb{E} \sum_{j=1}^{b} m_{*}\left(t-X_{j}\right), \quad t \geq 0 .
$$

Let $\mu_{j}$ be the distribution of $X_{j}$ on $(0, \infty)$; this is a measure of mass $1-\mathbb{P}\left(V_{j}=0\right)$; let further $\mu:=\sum_{j=1}^{b} \mu_{j}$. Then (3.10) can be written

$$
m_{*}(t)=1+\sum_{j=1}^{b} \mu_{j} * m_{*}(t)=1+\mu * m_{*}(t), \quad t \geq 0,
$$

where $\mu * f(t)=\int_{0}^{\infty} f(t-x) d \mu(x)$. This is the standard renewal equation, except that $\mu$ is not a probability measure.

Similarly, conditioning on $X_{1}, \ldots, X_{b}$, for $t \geq 0$,

$$
\begin{aligned}
\mathbb{E}\left(\left(N_{*}(t)-m_{*}(t)\right)^{2} \mid X_{1}, \ldots, X_{b}\right) & \\
= & \mathbb{E}\left(\left[\sum_{j=1}^{b} N_{*}^{(j)}\left(t-X_{j}\right)+1-m_{*}(t)\right]^{2} \mid X_{1}, \ldots, X_{b}\right) \\
= & \operatorname{Var}\left(\sum_{j=1}^{b} N_{*}^{(j)}\left(t-X_{j}\right)+1-m_{*}(t) \mid X_{1}, \ldots, X_{b}\right) \\
& +\left(\sum_{j=1}^{b} m_{*}\left(t-X_{j}\right)+1-m_{*}(t)\right)^{2} \\
& =\sum_{j=1}^{b} \sigma_{*}^{2}\left(t-X_{j}\right)+\left(\sum_{j=1}^{b} m_{*}\left(t-X_{j}\right)-m_{*}(t)+1\right)^{2} .
\end{aligned}
$$


Taking the expectation we obtain

$$
\sigma_{*}^{2}(t)=\mathbb{E} \sum_{j=1}^{b} \sigma_{*}^{2}\left(t-X_{j}\right)+h(t)=\mu * \sigma_{*}^{2}(t)+h(t), \quad t \geq 0,
$$

where, recalling (3.10),

$$
\begin{aligned}
h(t) & :=\mathbb{E}\left(\sum_{j=1}^{b} m_{*}\left(t-X_{j}\right)-m_{*}(t)+1\right)^{2} \\
& =\mathbb{E}\left(\sum_{j=1}^{b} m_{*}\left(t-X_{j}\right)-m_{*}(t)\right)^{2}+2\left(\mathbb{E} \sum_{j=1}^{b} m_{*}\left(t-X_{j}\right)-m_{*}(t)\right)+1 \\
& =\mathbb{E}\left(\sum_{j=1}^{b} m_{*}\left(t-X_{j}\right)-m_{*}(t)\right)^{2}-1 .
\end{aligned}
$$

Both (3.11) and (3.12) are instances of the general renewal equation (3.14) below, and from renewal theory we get the following result. We say that a function on $[0, \infty)$ is locally bounded if it is bounded on every finite interval.

Lemma 3.6. Assume that $\mathbf{V}$ is non-lattice. Let $f$ be a locally bounded measurable function on $[0, \infty)$. Then the renewal equation

$$
F=f+\mu * F
$$

has a unique locally bounded solution $F$ on $[0, \infty)$. If further $\mathbf{V}$ is non-lattice, then we have the following asymptotical results, as $t \rightarrow \infty$,

(i) If $f$ is a.e. continuous and $\int_{0}^{\infty} f^{*}(t) d t<\infty$, where $f^{*}(t):=$ $\sup _{u \geq t} e^{-u}|f(u)|$, then $F(t)=(\gamma+o(1)) e^{t}$, with $\gamma=\alpha^{-1} \int_{0}^{\infty} f(t) e^{-t} d t$.

(ii) If $f(t)=e^{t}$, then $F(t) \sim \alpha^{-1} t e^{t}$.

(iii) If $f(t)=e^{\lambda t}$ with $\operatorname{Re} \lambda=1$ and $\operatorname{Im} \lambda \neq 0$, then $F(t)=o\left(t e^{t}\right)$.

(iv) If $f(t)=e^{\lambda t}$ with $\operatorname{Re} \lambda>1$, then $F(t) \sim(1-\phi(\lambda))^{-1} e^{\lambda t}$.

Proof. For a function $f$ on $(0, \infty)$ and $z \in \mathbb{C}$, we define, when the integral exists, the Laplace transform $\tilde{f}(z):=\int_{0}^{\infty} e^{-z t} f(t) d t$. Similarly, the Laplace transform of $\mu$ is

$$
\widetilde{\mu}(z):=\int_{0}^{\infty} e^{-t z} d \mu(t)=\sum_{j=1}^{b} \mathbb{E} e^{-z X_{j}}=\sum_{j=1}^{b} \mathbb{E} V_{j}^{z}=\phi(z),
$$

at least for $\operatorname{Re} z \geq 0$.

Since $\mu$ is not a probability measure, we define another ("conjugate" or "tilted") measure $\nu$ on $[0, \infty)$ by

$$
d \nu(x)=e^{-x} d \mu(x)
$$


Then $\nu$ is a probability measure because, by (1.1),

$$
\nu[0, \infty)=\int_{0}^{\infty} e^{-x} d \mu(x)=\sum_{j=1}^{b} \int_{0}^{\infty} e^{-x} d \mu_{j}(x)=\sum_{j=1}^{b} \mathbb{E} e^{-X_{j}}=\sum_{j=1}^{b} \mathbb{E} V_{j}=1 .
$$

Further, the mean of the distribution $\nu$ is

$$
\mathbb{E} \nu=\int_{0}^{\infty} x d \nu(x)=\int_{0}^{\infty} x e^{-x} d \mu(x)=\sum_{j=1}^{b} \mathbb{E}\left(X_{j} e^{-X_{j}}\right)=\sum_{j=1}^{b} \mathbb{E}\left(\left(-\ln V_{j}\right) V_{j}\right)=\alpha
$$

and the Laplace transform is, for $\operatorname{Re} z \geq 0$, recalling (3.15),

$$
\widetilde{\nu}(z):=\int_{0}^{\infty} e^{-z x} d \nu(x)=\int_{0}^{\infty} e^{-x-z x} d \mu(x)=\widetilde{\mu}(z+1)=\phi(z+1) .
$$

Let $g(t):=e^{-t} f(t)$ and $G(t):=e^{-t} F(t)$. Then (3.14) translates to

$$
\begin{aligned}
G(t) & =e^{-t} F(t)=e^{-t} f(t)+\int_{0}^{\infty} e^{-t} F(t-x) d \mu(x) \\
& =g(t)+\int_{0}^{\infty} G(t-x) e^{-x} d \mu(x)=g(t)+\nu * G(t) .
\end{aligned}
$$

In other words, $G$ satisfies the renewal equation for the probability measure $\nu$, so we can use standard results from renewal theory.

First, it is well known that the equation $G=g+\nu * G$ has a unique locally bounded solution which is given by $G=\sum_{n=0}^{\infty} \nu^{* n} * g$, and thus $F=\sum_{n=0}^{\infty} \mu^{* n} * f$; see e.g. [1, Theorem IV.2.4] (which also applies directly to $F$ ). If we let $Y_{1}, Y_{2}, \ldots$ be i.i.d. random variables with the distribution $\nu$, and let $S_{n}:=\sum_{1}^{n} Y_{i}$, this can be written

$$
G(t)=\sum_{n=0}^{\infty} \mathbb{E}\left(g\left(t-S_{n}\right) \mathbf{1}_{\left\{S_{n} \leq t\right\}}\right)=\mathbb{E} \sum_{S_{n} \leq t} g\left(t-S_{n}\right) .
$$

Under the assumptions of (i), $f^{*}$ is non-increasing and integrable; further, $\sup f^{*} \leq \sup _{[0,1]}|f|+f^{*}(1)<\infty$, so $f^{*}$ is bounded too. Hence [1, Proposition IV.4.1(v),(iv)] shows that $f^{*}$ and $g$ are directly Riemann integrable. The key renewal theorem [1, Theorem IV.4.3] and (3.17) now yield $G(t) \rightarrow \alpha^{-1} \int_{0}^{\infty} g(x) d x=\gamma$, which proves (i).

In case (ii) we have $g(t)=1$, and thus $G(t) \sim \alpha^{-1} t$ by the elementary renewal theorem 1, IV.(1.5) and Theorem 2.4].

For (iii), $g(t)=e^{(\lambda-1) t}=e^{i b t}$ for some real $b \neq 0$. The solution to (3.14) may be written [1, Theorem IV.2.4] $G(t)=\int_{0}^{t} g(t-x) d U(x)$, where $U$ is the locally bounded solution to $U=1+\nu * U$ (i.e., $U=G$ for case (ii)). Since, in analogy with (3.17), $\int x^{2} d \nu(x)=\sum_{j} \mathbb{E}\left(\left(\ln V_{j}\right)^{2} V_{j}\right)<\infty$, the distribution $\nu$ has finite variance, and the renewal theorem has the sharper version [9, Theorem XI.3.1]

$$
U(t)=\alpha^{-1} t+c+R(t)
$$


where $c$ is a certain constant $\left(\int x^{2} d \nu / 2 \alpha^{2}\right)$ and $R(t) \rightarrow 0$ as $t \rightarrow \infty$. Hence, using integration by parts for one term,

$$
\begin{aligned}
G(t) & =\int_{0}^{t} e^{\mathrm{i} b(t-x)} \alpha^{-1} d x+c e^{\mathrm{i} b t}+\int_{0}^{t} e^{\mathrm{i} b(t-x)} d R(x) \\
& =O(1)+R(t)-R(0) e^{\mathrm{i} b t}+\mathrm{i} b \int_{0}^{t} e^{\mathrm{i} b(t-x)} R(x) d x=o(t) .
\end{aligned}
$$

For (iv), we have by (3.19) with $g(t)=e^{(\lambda-1) t}$, using dominated convergence and (3.18),

$$
\begin{aligned}
e^{-\lambda t} F(t) & =e^{(1-\lambda) t} G(t)=\sum_{n=0}^{\infty} \mathbb{E}\left(e^{(1-\lambda) t+(\lambda-1)\left(t-S_{n}\right)} \mathbf{1}_{\left\{t \leq S_{n}\right\}}\right) \\
& \rightarrow \sum_{n=0}^{\infty} \mathbb{E}\left(e^{-(\lambda-1) S_{n}}\right)=\sum_{n=0}^{\infty} \mathbb{E}\left(e^{-(\lambda-1) Y_{1}}\right)^{n}=\sum_{n=0}^{\infty} \widetilde{\nu}(\lambda-1)^{n} \\
& =\sum_{n=0}^{\infty} \phi(\lambda)^{n}=(1-\phi(\lambda))^{-1} .
\end{aligned}
$$

Proof of Theorem 3.1. We first apply Lemma 3.6(i) to (3.11), with $f(t)=1$ for $t \geq 0$, and obtain $\gamma=\alpha^{-1}$ and $m_{*}(t) \sim \alpha^{-1} e^{t}$, which proves Theorem 3.1(i).

To obtain more refined asymptotics, we use Laplace transforms. Let $H(t):=$ $\mathbf{1}_{\{t \geq 0\}}$ (the Heaviside function), and note that $\widetilde{H}(z)=\int_{0}^{\infty} e^{-t z} d t=1 / z, \operatorname{Re} z>0$. Since the Laplace transform converts convolutions to products, the renewal equation (3.11) yields $\widetilde{m_{*}}(z)=\widetilde{H}(z)+\widetilde{\mu}(z) \widetilde{m_{*}}(z)$, and thus

$$
\widetilde{m_{*}}(z)=\frac{\widetilde{H}(z)}{1-\widetilde{\mu}(z)}=\frac{1}{z(1-\phi(z))},
$$

for $z$ such that the transforms exist. By the estimate $m_{*}(t) \sim \alpha^{-1} e^{t}$ above, $m_{*}(t)=$ $O\left(e^{t}\right)$ and thus $\widetilde{m_{*}}(z)$ exists for $\operatorname{Re} z>1$. Consequently, (3.20) holds for $\operatorname{Re} z>1$, and can be used to extend $\widetilde{m_{*}}(z)$ to a meromorphic function for $\operatorname{Re} z>0$.

We want to invert the Laplace transform in (3.20). This is simple if $\phi$ is rational, yielding (3.6). (Note that $\phi(0)=\mathbb{E}\left|\left\{j: V_{j}>0\right\}\right|>1$.) In general, there are difficulties to doing this directly, because $\widetilde{m_{*}}(z)$ is not integrable along a vertical line $\operatorname{Re} z=s$; it decreases too slowly as $|\operatorname{Im} z| \rightarrow \infty$. We therefore regularize. Let $\varepsilon>0$, and let $H_{\varepsilon}:=H * \varepsilon^{-1} \mathbf{1}_{[0, \varepsilon]} ;$ thus

$$
H_{\varepsilon}(t)= \begin{cases}0, & t<0 \\ 1-t / \varepsilon, & 0 \leq t<\varepsilon \\ 1, & t \geq \varepsilon\end{cases}
$$

Let $m_{* \varepsilon}=\sum_{n=0}^{\infty} \mu^{* n} * H_{\varepsilon}$ be the locally bounded solution to $m_{* \varepsilon}=H_{\varepsilon}+\mu * m_{* \varepsilon}$. Note that $H_{\varepsilon}(t) \leq H(t) \leq H_{\varepsilon}(t+\varepsilon)$, and thus

$$
m_{* \varepsilon}(t) \leq m_{*}(t) \leq m_{* \varepsilon}(t+\varepsilon) .
$$


We have

$$
\widetilde{H}_{\varepsilon}(z)=\widetilde{H}(z) \varepsilon^{-1} \int_{0}^{\varepsilon} e^{-z t} d t=\frac{1-e^{-\varepsilon z}}{\varepsilon z^{2}}, \quad \operatorname{Re} z>0,
$$

and we find, arguing as for (3.20) above,

$$
\widetilde{m_{* \varepsilon}}(z)=\frac{\widetilde{H_{\varepsilon}}(z)}{1-\widetilde{\mu}(z)}=\frac{1-e^{-\varepsilon z}}{\varepsilon z^{2}(1-\phi(z))},
$$

first for $\operatorname{Re} z>1$, and then for $\operatorname{Re} z>0$, extending $\widetilde{m_{* \varepsilon}}$ to a meromorphic function in this domain. This function decreases (using Condition $\mathrm{B}(\delta)$ and Lemma 2.1) as $|\operatorname{Im} z|^{-2}$ on vertical lines $\operatorname{Re} z=s \geq \delta$, and is thus integrable there. Hence, the Laplace inversion formula (a Fourier inversion) shows that for any $s>1$ and $t \geq 0$,

$$
m_{* \varepsilon}(t)=\frac{1}{2 \pi \mathrm{i}} \int_{s-\mathrm{i} \infty}^{s+\mathrm{i} \infty} e^{t z} \widetilde{m_{* \varepsilon}}(z) d z
$$

We may, increasing $\delta$ a little if necessary, assume that $\phi(z)=1$ has no roots with $\operatorname{Re} z=\delta$; in cases (iii), (iv) and (v) we may similarly assume that each $\lambda \in \Lambda$ with $\operatorname{Re} \lambda>\delta$ has $\phi^{\prime}(\lambda) \neq 0$. It is then easy to show, using Condition $\mathrm{B}(\delta)$ and Lemma 2.1. that we may shift the line of integration in (3.22) to $\operatorname{Re} z=\delta$ and obtain, for $0<\varepsilon \leq 1$,

$$
\begin{aligned}
m_{* \varepsilon}(t) & =\frac{1}{2 \pi \mathrm{i}} \int_{\delta-\mathrm{i} \infty}^{\delta+\mathrm{i} \infty} e^{t z \widetilde{m_{* \varepsilon}}}(z) d z+\sum_{i=1}^{M} \operatorname{Res}_{z=\lambda_{i}}\left(e^{t z \widetilde{m_{* \varepsilon}}}(z)\right) \\
& =O\left(e^{t \delta} \int_{\delta-\mathrm{i} \infty}^{\delta+\mathrm{i} \infty} \min \left(\frac{1}{|z|}, \frac{\varepsilon}{|z|^{2}}\right)|d z|\right)+\sum_{i=1}^{M} \frac{e^{t \lambda_{i}}}{-\lambda_{i} \phi^{\prime}\left(\lambda_{i}\right)} \frac{1-e^{-\varepsilon \lambda_{i}}}{\varepsilon \lambda_{i}} \\
& =\sum_{i=1}^{M} \frac{e^{t \lambda_{i}}}{-\lambda_{i} \phi^{\prime}\left(\lambda_{i}\right)}(1+O(\varepsilon))+O\left(e^{t \delta}\left(1+\ln \frac{1}{\varepsilon}\right)\right)
\end{aligned}
$$

Now choose $\varepsilon:=e^{-t}$; we then obtain

$$
m_{* \varepsilon}(t)=\sum_{i=1}^{M} \frac{e^{t \lambda_{i}}}{-\lambda_{i} \phi^{\prime}\left(\lambda_{i}\right)}+O(1)+O\left(e^{t \delta}(1+t)\right), \quad t \geq 0 .
$$

Replacing $t$ by $t+\varepsilon$, we obtain the same estimate for $m_{* \varepsilon}(t+\varepsilon)$, and thus (3.21) yields

$$
m_{*}(t)=\sum_{i=1}^{M} \frac{e^{t \lambda_{i}}}{-\lambda_{i} \phi^{\prime}\left(\lambda_{i}\right)}+O\left(e^{t \delta^{\prime}}\right), \quad t \geq 0,
$$

which yields Theorem 3.1(ii).

For the estimates of the variance, we use Lemma 3.6 and (3.12). It is easily seen (by dominated convergence) that $h$ in (3.13) is a.e. continuous. Choose $\delta^{\prime}>\delta$ with $\delta<\delta^{\prime}<\operatorname{Re} \lambda_{M}$; in case (iii) with $M=1$, let further $\delta^{\prime}<1 / 2$. 
Note that then (3.23) trivially holds for $t<0$ too. Hence,

$$
\begin{aligned}
\sum_{j=1}^{b} m_{*}\left(t-X_{j}\right)-m_{*}(t) & =\sum_{i=1}^{M} \frac{\sum_{j=1}^{b} e^{\left(t-X_{j}\right) \lambda_{i}}-e^{t \lambda_{i}}}{-\lambda_{i} \phi^{\prime}\left(\lambda_{i}\right)}+O\left(e^{t \delta^{\prime}}\right) \\
& =\sum_{i=2}^{M} \frac{e^{t \lambda_{i}}}{-\lambda_{i} \phi^{\prime}\left(\lambda_{i}\right)}\left(\sum_{j=1}^{b} V_{j}^{\lambda_{i}}-1\right)+O\left(e^{t \delta^{\prime}}\right),
\end{aligned}
$$

where we use the fact that $\lambda_{1}=1$ and thus $\sum_{j=1}^{b} V_{j}^{\lambda_{1}}-1=\sum_{j=1}^{b} V_{j}-1=0$. Consequently, by (3.13) and (2.1), letting $\sigma_{2}:=\operatorname{Re} \lambda_{2}>\delta^{\prime}$ if $M \geq 2$, and $\sigma_{2}:=\delta^{\prime}$ if $M=1$,

$$
h(t)=\sum_{i=2}^{M} \sum_{k=2}^{M} \frac{e^{\left(\lambda_{i}+\lambda_{k}\right) t}}{\lambda_{i} \lambda_{k} \phi^{\prime}\left(\lambda_{i}\right) \phi^{\prime}\left(\lambda_{k}\right)} \psi\left(\lambda_{i}, \lambda_{k}\right)+O\left(e^{t\left(\delta^{\prime}+\sigma_{2}\right)}\right) .
$$

For Theorem 3.1(iii), (3.24) yields $h(t)=O\left(e^{2 \sigma_{2} t}\right)$ with $\sigma_{2}<1 / 2$, and Lemma 3.6)(i) applies to (3.12), yielding $\sigma_{*}^{2}(t) \sim \gamma e^{t}$. We postpone the calculation of $\beta=\gamma$, verifying (3.4), to Lemma 3.7

In Theorem 3.1(iv) and (v), we treat the terms in (3.24) separately, using linearity; for the error term we also use monotonicity and comparison with the case $f(t)=$ $e^{t\left(\delta^{\prime}+\sigma_{2}\right)}$. The results follow by applying Lemma 3.6(i)-(iv) to (3.12), letting $t:=$ $\ln x$, with the leading terms coming from the case $\operatorname{Re} \lambda_{k}=\operatorname{Re} \lambda_{i}=\operatorname{Re} \lambda_{2}$, and for (iv) further $\lambda_{k}=\overline{\lambda_{i}}$.

Furthermore, by (3.5), $\beta=0$ in (iv) only if for every $\lambda_{i} \in \Lambda(1 / 2)$, we have $\psi\left(\lambda_{i}, \bar{\lambda}_{i}\right)=\mathbb{E}\left|\sum_{j} V_{j}^{\lambda_{i}}-\phi\left(\lambda_{i}\right)\right|^{2}=0$, and thus (1.8) holds.

Lemma 3.7. If $\operatorname{Re} \lambda_{2}<1 / 2$ or $M=1$, then, with $h(t)$ as in (3.13),

$$
\int_{0}^{\infty} h(t) e^{-t} d t=\frac{1}{2 \pi} \int_{-\infty}^{\infty} \frac{\psi(1 / 2+\mathrm{i} u, 1 / 2-\mathrm{i} u)}{|1 / 2+\mathrm{i} u|^{2}|1-\phi(1 / 2+\mathrm{i} u)|^{2}} d u>0 .
$$

Proof. Write $f(t):=\left(m_{*}(t)-\alpha^{-1} e^{t}\right) e^{-t / 2},-\infty<t<\infty$. Thus, by (3.23), $f(t)=O\left(e^{-\left(1 / 2-\sigma_{2}\right) t}\right)$ for $t \geq 0$ and $f(t)=-\alpha^{-1} e^{t / 2}=O\left(e^{-|t| / 2}\right)$ for $t<0$. In particular, $f \in L^{2}(-\infty, \infty)$. Furthermore, the (two-sided) Laplace transform $\tilde{f}(z):=\int_{-\infty}^{\infty} f(t) e^{-t z} d t$ is analytic for $-\left(1 / 2-\sigma_{2}\right)<\operatorname{Re} z<1 / 2$.

Define further $f_{1}(t):=f(t) e^{t / 2}=m_{*}(t)-\alpha^{-1} e^{t}$ and $f_{2}(t):=f_{1}(t) \mathbf{1}_{\{t \geq 0\}}$. Then $f_{2}(t)=O\left(e^{\sigma_{2} t}\right)$, and thus the Laplace transform $\tilde{f}_{2}(z)$ is analytic for $\operatorname{Re} z>\sigma_{2}$. For $\operatorname{Re} z>1$ we have, by (3.20),

$\tilde{f}_{2}(z)=\int_{0}^{\infty} e^{-t z}\left(m_{*}(t)-\alpha^{-1} e^{t}\right) d t=\widetilde{m_{*}}(z)-\alpha^{-1}(z-1)^{-1}=\frac{1}{z(1-\phi(z)}-\frac{1}{\alpha(z-1)} ;$

by analytic continuation, this formula holds for $\operatorname{Re} z>\sigma_{2}$. Consequently, for $\sigma_{2}<$ $\operatorname{Re} z<1$,

$$
\tilde{f}_{1}(z)=\tilde{f}_{2}(z)+\int_{-\infty}^{0} e^{-t z}\left(-\alpha^{-1} e^{t}\right) d t=\tilde{f}_{2}(z)-\alpha^{-1}(1-z)^{-1}=\frac{1}{z(1-\phi(z)} .
$$


Since $\tilde{f}(z)=\tilde{f}_{1}(z+1 / 2)$, we find the Fourier transform

$$
\hat{f}(u):=\int_{0}^{\infty} e^{-\mathrm{i} u t} f(t) d t=\tilde{f}(\mathrm{i} u)=\tilde{f}_{1}\left(\frac{1}{2}+\mathrm{i} u\right)=\frac{1}{\left(\frac{1}{2}+\mathrm{i} u\right)\left(1-\phi\left(\frac{1}{2}+\mathrm{i} u\right)\right)} .
$$

Next, since $\sum_{j} e^{-X_{j}}=\sum_{j} V_{j}=1$,

$$
\begin{aligned}
\sum_{j=1}^{b} m_{*}\left(t-X_{j}\right)-m_{*}(t) & =\sum_{j=1}^{b} f_{1}\left(t-X_{j}\right)-f_{1}(t)+\alpha^{-1} \sum_{j=1}^{b} e^{t-X_{j}}-\alpha^{-1} e^{t} \\
& =\sum_{j=1}^{b} f_{1}\left(t-X_{j}\right)-f_{1}(t),
\end{aligned}
$$

so by (3.13), and defining $\Psi(x, y):=\int_{-\infty}^{\infty} f(t-x) f(t-y) d t$,

$$
\begin{aligned}
\int_{0}^{\infty} h(t) e^{-t} d t+1 & =\int_{0}^{\infty}(h(t)+1) e^{-t} d t=\mathbb{E} \int_{-\infty}^{\infty}\left(\sum_{j=1}^{b} f_{1}\left(t-X_{j}\right)-f_{1}(t)\right)^{2} e^{-t} d t \\
& =\mathbb{E} \int_{-\infty}^{\infty}\left|\sum_{j=1}^{b} e^{-X_{j} / 2} f\left(t-X_{j}\right)-f(t)\right|^{2} d t \\
& =\mathbb{E} \sum_{j, k=1}^{b} e^{-X_{j} / 2-X_{k} / 2} \Psi\left(X_{j}, X_{k}\right)-2 \mathbb{E} \sum_{j=1}^{b} e^{-X_{j} / 2} \Psi\left(X_{j}, 0\right)+\Psi(0,0) .
\end{aligned}
$$

By Parseval's relation and $f=\bar{f}$,

$$
\Psi(x, y)=\frac{1}{2 \pi} \int_{-\infty}^{\infty} e^{-\mathrm{i} u x} \hat{f}(u) \overline{e^{-\mathrm{i} u y} \hat{f}(u)} d u \frac{1}{2 \pi} \int_{-\infty}^{\infty}|\hat{f}(u)|^{2} e^{\mathrm{i} u(y-x)} d u .
$$

Hence,

$$
\begin{aligned}
\int_{0}^{\infty} h(t) e^{-t} d t+1=\mathbb{E} \frac{1}{2 \pi} \int_{-\infty}^{\infty}|\hat{f}(u)|^{2}\left(\sum_{j, k=1}^{b} e^{-X_{j} / 2-X_{k} / 2+\mathrm{i} u\left(X_{k}-X_{j}\right)}\right. \\
\left.\quad-\sum_{j=1}^{b} e^{-X_{j} / 2+\mathrm{i} u X_{j}}-\sum_{k=1}^{b} e^{-X_{k} / 2-\mathrm{i} u X_{k}}+1\right) d u \\
=\mathbb{E} \frac{1}{2 \pi} \int_{-\infty}^{\infty}|\hat{f}(u)|^{2} \mathbb{E}\left|\sum_{j=1}^{b} V_{j}^{1 / 2-\mathrm{i} u}-1\right|^{2} d u \\
=\mathbb{E} \frac{1}{2 \pi} \int_{-\infty}^{\infty}|\hat{f}(u)|^{2}\left(\psi(1 / 2+\mathrm{i} u, 1 / 2-\mathrm{i} u)+|\phi(1 / 2+\mathrm{i} u)-1|^{2}\right) d u
\end{aligned}
$$

Using (3.25),

$$
\mathbb{E} \frac{1}{2 \pi} \int_{-\infty}^{\infty}|\hat{f}(u)|^{2}|\phi(1 / 2+\mathrm{i} u)-1|^{2} d u=\int_{-\infty}^{\infty} \frac{d u}{|1 / 2+\mathrm{i} u|^{2}}=\int_{-\infty}^{\infty} \frac{d u}{\frac{1}{4}+u^{2}}=2 \pi,
$$

and the formula follows by (3.25). 
Since $\psi(z, \bar{z}) \geq 0$, and by dominated convergence is continuous for $\operatorname{Re} z \geq 0$, it follows from (3.4) that $\beta=0$ only if for every $z$ with $\operatorname{Re} z=1 / 2, \psi(z, \bar{z})=$ $\mathbb{E}\left|\sum_{j} V_{j}^{z}-\phi(z)\right|^{2}=0$, and thus

$$
\sum_{j=1}^{b} V_{j}^{1 / 2+\mathrm{i} u}=\phi(1 / 2+\mathrm{i} u)
$$

a.s., for every real $u$. Considering first rational $u$, we see that a.s. (3.26) holds for all real $u$.

However, for any realization $\left(V_{1}, \ldots, V_{b}\right)$ and $\varepsilon>0$, it is by Dirichlet's theorem 14. Theorem 201] possible to find arbitrarily large $u$ with $\operatorname{Re} V_{j}^{1 / 2+\mathrm{i} u} \geq(1-\varepsilon) V_{j}^{1 / 2}$ for $j=1, \ldots, b$, and thus (3.26) implies that $\lim _{\sup } \operatorname{su}_{u \rightarrow \infty}\left|\phi\left(\frac{1}{2}+\mathrm{i} u\right)\right| \geq \phi(1 / 2) \geq 1$. This contradicts Condition $\mathrm{B}(\delta)$ and Lemma 2.1. Hence $\beta>0$.

Proof of Theorem 3.4. (i): As remarked above, (3.6) follows by inverting the Laplace transform in (3.20), using a partial fraction expansion.

(ii): Note first that $\phi(z) \rightarrow 0$ as $z \rightarrow+\infty$ (by dominated convergence), and thus, $\phi$ being rational, $\phi(\infty)=0$ and $\phi(z) \rightarrow 0$ as $|z| \rightarrow \infty$; hence Condition $\mathrm{B}(\delta)$ holds for every $\delta$. We thus see that the conditions of Theorem 3.1(iii) are satisfied, and from the proof above we see that, with $h$ given by (3.13),

$$
\beta=\alpha^{-1} \int_{0}^{\infty} h(t) e^{-t} d t=\alpha^{-1} \int_{1}^{\infty} h(\ln x) x^{-2} d x
$$

We have, by (3.6), $m(x)=\sum_{i=0}^{M} a_{i} x^{\lambda_{i}} \mathbf{1}_{\{x \geq 1\}}$ and thus

$$
m_{*}\left(\ln x-X_{j}\right)=m\left(x e^{-X_{j}}\right)=m\left(x V_{j}\right)=\sum_{i=0}^{M} a_{i}\left(x V_{j}\right)^{\lambda_{i}} \mathbf{1}_{\left\{x \geq V_{j}^{-1}\right\}} .
$$

Hence, letting $V_{0}:=1, \varepsilon_{0}=-1$ and $\varepsilon_{j}=1$ for $j \geq 1$, and recalling (1.1),

$$
\begin{aligned}
H(x) & :=\sum_{j=1}^{b} m_{*}\left(\ln x-X_{j}\right)-m_{*}(\ln x)=\sum_{i=0}^{M} a_{i} x^{\lambda_{i}}\left(\sum_{j=1}^{b} V_{j}^{\lambda_{i}} \mathbf{1}_{\left\{x \geq V_{j}^{-1}\right\}}-\mathbf{1}_{\{x \geq 1\}}\right) \\
& =\sum_{i \neq 1} a_{i} x^{\lambda_{i}} \sum_{j=0}^{b} V_{j}^{\lambda_{i}} \varepsilon_{j} \mathbf{1}_{\left\{x \geq V_{j}^{-1}\right\}}-a_{1} x \sum_{j=1}^{b} V_{j} \mathbf{1}_{\left\{1 \leq x<V_{j}^{-1}\right\}} .
\end{aligned}
$$


By (3.13), this leads to

$$
\begin{gathered}
\int_{1}^{\infty} h(\ln x) x^{-2} d x+1=\int_{1}^{\infty} \mathbb{E} H(x)^{2} x^{-2} d x=\mathbb{E} \int_{1}^{\infty} H(x)^{2} x^{-2} d x \\
=\mathbb{E} \sum_{i, k \neq 1} a_{i} a_{k} \sum_{j, l=0}^{b} \varepsilon_{j} \varepsilon_{l} V_{j}^{\lambda_{i}} V_{l}^{\lambda_{k}} \int_{V_{j}^{-1} \vee V_{l}^{-1}}^{\infty} x^{\lambda_{i}+\lambda_{k}-2} d x \\
-2 \mathbb{E} \sum_{i \neq 1} a_{1} a_{i} \sum_{j=0}^{b} \sum_{l=1}^{b} \varepsilon_{j} V_{j}^{\lambda_{i}} V_{l} \int_{V_{j}^{-1}}^{V_{l}^{-1}} x^{\lambda_{i}-1} d x \mathbf{1}_{\left\{V_{j}^{-1} \leq V_{l}^{-1}\right\}} \\
+\mathbb{E} a_{1}^{2} \sum_{j, l=1}^{b} V_{j} V_{l} \int_{1}^{V_{j}^{-1} \wedge V_{l}^{-1}} d x
\end{gathered}
$$

and the result follows by straightforward calculations, noting that $a_{1}=\alpha^{-1}$.

\section{Zolotarev MetriC AND Minimal $L_{s}$ MetriC}

In this section we collect properties of the minimal $L_{s}$ metric and the Zolotarev metric that are used subsequently.

We denote by $\mathcal{M}^{d}$ the space of probability measures on $\mathbb{R}^{d}$. The minimal $L_{s}$ metric $\ell_{s}, s>0$, is defined on the subspace $\mathcal{M}_{s}^{d} \subset \mathcal{M}^{d}$ of probability measures with finite absolute moment of order $s$ by

$$
\ell_{s}(\mu, \nu):=\inf \left\{\|X-Y\|_{s}^{s \wedge 1}: X \stackrel{d}{=} \mu, Y \stackrel{d}{=} \nu\right\}, \quad \mu, \nu \in \mathcal{M}_{s}^{d}
$$

where $\|X\|_{s}:=\left(\mathbb{E}|X|^{s}\right)^{1 / s}$ denotes the $L_{s}$ norm of $X$. The infimum is taken over all random vectors of $X, Y$ on a joint probability space with the given marginal distributions $\mu$ and $\nu$. (In other words, over all couplings $(X, Y)$ of $\mu$ and $\nu$.) We will also use the notation $\ell_{s}(X, Y):=\ell_{s}(\mathcal{L}(X), \mathcal{L}(Y))$.

For $s \geq 1$ and $\gamma \in \mathbb{R}^{d}$, we denote by $\mathcal{M}_{s}^{d}(\gamma) \subset \mathcal{M}_{s}^{d}$ the subspace of probability measures with expectation $\gamma$. The pairs $\left(\mathcal{M}_{s}^{d}, \ell_{s}\right), s>0$, and $\left(\mathcal{M}_{s}^{d}(\gamma), \ell_{s}\right), s \geq 1$, are complete metric spaces and convergence in $\ell_{s}$ is equivalent to weak convergence plus convergence of the absolute moments of order $s$.

Random vectors $(X, Y)$ with $X \stackrel{d}{=} \mu, Y \stackrel{d}{=} \nu$, and $\ell_{s}(\mu, \nu)=\|X-Y\|_{s}^{s \wedge 1}$ are called optimal couplings of $(\mu, \nu)$. Such optimal couplings exist for all $\mu, \nu \in \mathcal{M}_{s}^{d}$. These properties can be found in Dall'Aglio 6], Major [23], Bickel and Freedman [2], and Rachev [25]. Similar properties hold for probability measures on $\mathbb{C}^{d}$ (because $\mathbb{C}^{d} \cong \mathbb{R}^{2 d}$ ), where we use corresponding notations.

The Zolotarev metric $\zeta_{s}, s>0$ is defined by

$$
\zeta_{s}(X, Y):=\zeta(\mathcal{L}(X), \mathcal{L}(Y)):=\sup _{f \in \mathcal{F}_{s}}|\mathbb{E}(f(X)-f(Y))|
$$

where $s=m+\alpha$ with $0<\alpha \leq 1, m=\lceil s\rceil-1 \geq 0$ is an integer, and

$$
\mathcal{F}_{s}:=\left\{f \in C^{m}\left(\mathbb{R}^{d}, \mathbb{R}\right):\left\|f^{(m)}(x)-f^{(m)}(y)\right\| \leq\|x-y\|^{\alpha}\right\},
$$


where $C^{m}\left(\mathbb{R}^{d}, \mathbb{R}\right)$ denotes the space of $m$ times continuously differentiable functions $f$ on $\mathbb{R}^{d}$ and $f^{(m)}$ their $m$ th derivative.

The expression $\zeta_{s}(X, Y)$ is finite if $X$ and $Y$ have finite absolute moments of order $s$ and all mixed moments of orders $1, \ldots, m$ of $X$ and $Y$ coincide.

The metric $\zeta_{s}$ is ideal of order $s$, i.e., we have for $Z$ independent of $(X, Y)$ and any $d \times d$ square matrix $A$

$$
\zeta_{s}(X+Z, Y+Z) \leq \zeta_{s}(X, Y), \quad \zeta_{s}(A X, A Y) \leq\|A\|_{\mathrm{op}}^{s} \zeta_{s}(X, Y),
$$

where $\|A\|_{\text {op }}:=\sup _{\|u\|=1}\|A u\|$ denotes the operator norm of the matrix. Convergence in $\zeta_{s}$ implies weak convergence. For general reference and properties of $\zeta_{s}$ we refer to Zolotarev [29, 30] and Rachev [25].

\section{General Contraction theorems in Continuous time}

In this section we extend a general contraction theorem for recursive sequences $\left(Y_{n}\right)_{n \geq 0}$ of $d$-dimensional vectors as developed in Neininger and Rüschendorf [24] to families $\left(Y_{t}\right)_{t \geq 0}$ of $d$-dimensional vectors with continuous parameter $t \in[0, \infty)$. For this, we assume that we have

$$
Y_{t} \stackrel{d}{=} \sum_{r=1}^{K} A_{r}(t) Y_{T_{r}^{(t)}}^{(r)}+b_{t}, \quad t \geq \tau_{0}
$$

where $K$ is a positive integer and $\tau_{0} \geq 0$, and we have $\left(Y_{t}^{(1)}\right)_{t}, \ldots,\left(Y_{t}^{(K)}\right)_{t}$, $\left(A_{1}(t), \ldots, A_{K}(t), b_{t}, T^{(t)}\right)_{t}$ independent, where $T^{(t)}=\left(T_{1}^{(t)}, \ldots, T_{K}^{(t)}\right)$ is a vector of random indices $T_{r}^{(t)} \in[0, t]$, the $A_{r}(t)$ are random $d \times d$ matrices for $r=1, \ldots, K$ and $b_{t}$ is a random $d$ dimensional vector. Finally, in (5.1), we have that for each $t \geq 0, Y_{t}$ and $Y_{t}^{(r)}$ are identically distributed for all $r=1, \ldots, K$.

We assume that all $Y_{t}$ as well as $A_{r}(t), b_{t}$ and $T^{(t)}$ are defined on some probability space $(\Omega, \mathcal{F}, \mu)$, and that they are measurable functions of $(t, \omega)$. (This is a technicality to ensure that the sum in (5.1) is well-defined. Note, however, that the joint distribution of $Y_{t}$ for different $t$ is irrelevant.)

We introduce the normalized random vectors

$$
X_{t}:=C_{t}^{-1 / 2}\left(Y_{t}-M_{t}\right), \quad t \geq 0,
$$

where $M_{t} \in \mathbb{R}^{d}$ and $C_{t}$ is a symmetric, positive definite square matrix. We assume that $M_{t}$ and $C_{t}$ are measurable functions of $t$; further restrictions on $M_{t}$ and $C_{t}$ will be given in Convention C. The recurrence (5.1) implies a recurrence for $X_{t}$,

$$
X_{t} \stackrel{d}{=} \sum_{r=1}^{K} A_{r}^{(t)} X_{T_{r}^{(t)}}^{(r)}+b^{(t)}, \quad t \geq \tau_{0}
$$


with independence relations as in (5.1) and

$$
A_{r}^{(t)}=C_{t}^{-1 / 2} A_{r}(t) C_{T_{r}^{(t)}}^{1 / 2}, \quad b^{(t)}=C_{t}^{-1 / 2}\left(b_{t}-M_{t}+\sum_{r=1}^{K}\left(A_{r}(t) M_{T_{r}^{(t)}}\right)\right) .
$$

As for the case with integer indexed vectors we establish a transfer theorem of the following form: Appropriate convergence of the coefficients $A_{r}^{(t)} \rightarrow A_{r}^{*}, b^{(t)} \rightarrow b^{*}$ implies weak convergence of the quantities $X_{t}$ to a limit $X$. The distribution $\mathcal{L}(X)$ of $X$ is a fixed point of the limiting equation obtained from (5.3) by letting formally $t \rightarrow \infty$ :

$$
X \stackrel{d}{=} \sum_{r=1}^{K} A_{r}^{*} X^{(r)}+b^{*}
$$

where $\left(A_{1}^{*}, \ldots, A_{K}^{*}, b^{*}\right), X^{(1)}, \ldots, X^{(K)}$ are independent and $X^{(r)} \stackrel{d}{=} X$ for $r=$ $1, \ldots, K$. To formalize this we introduce the map $T$ on the space $\mathcal{M}^{d}$ of probability measures on $\mathbb{R}^{d}$ by

$$
T: \mathcal{M}^{d} \rightarrow \mathcal{M}^{d}, \quad \eta \mapsto \mathcal{L}\left(\sum_{r=1}^{K} A_{r}^{*} Z^{(r)}+b^{*}\right),
$$

where $\left(A_{1}^{*}, \ldots, A_{K}^{*}, b^{*}\right), Z^{(1)}, \ldots, Z^{(K)}$ are independent and $\mathcal{L}\left(Z^{(r)}\right)=\eta$ for $r=$ $1, \ldots, K$. Then $X$ is a solution of (5.5) if and only if $\mathcal{L}(X)$ is a fixed point of $T$.

We make use of Zolotarev's metric $\zeta_{s}$ with $0<s \leq 3$. To ensure finiteness of the metric subsequently we make the following assumptions about the scaling imposed in (5.2):

Convention C. For $1<s \leq 3$ we assume that $M_{t}=\mathbb{E} Y_{t}$. For $2<s \leq 3$ we assume that $\operatorname{Cov}\left(Y_{t}\right)$ is positive definite for all $t \geq \tau_{1}$ with a $\tau_{1} \geq \tau_{0}$ and that $C_{t}=\operatorname{Id}_{d}$ for $0 \leq t<\tau_{1}$ and $C_{t}=\operatorname{Cov}\left(Y_{t}\right)$ for $t \geq \tau_{1}$.

This convention implies that $X_{t}$ is centered for $1<s \leq 3$ and has $\operatorname{Id}_{d}$ as its covariance matrix for $2<s \leq 3$ and $t \geq \tau_{1}$. (For $0<s \leq 1$, Convention $\mathrm{C}$ is void.)

Theorem 5.1. Let $0<s \leq 3$ and let $\left(Y_{t}\right)_{t \geq 0}$ be a process of random vectors satisfying (5.1) such that $\left\|Y_{t}\right\|_{s}<\infty$ for every $t$. Denote by $X_{t}$ the rescaled quantities in (5.2), assuming Convention C. Assume that $\left\|A_{r}^{(t)}\right\|_{s}<\infty,\left\|b^{(t)}\right\|_{s}<\infty$ and $\sup _{0 \leq u \leq t}\left\|X_{u}\right\|_{s}<\infty$ for every $t \geq 0$, and

$$
\begin{aligned}
& \left(A_{1}^{(t)}, \ldots, A_{K}^{(t)}, b^{(t)}\right) \stackrel{\ell_{s}}{\longrightarrow}\left(A_{1}^{*}, \ldots, A_{K}^{*}, b^{*}\right), \\
& \mathbb{E} \sum_{r=1}^{K}\left\|A_{r}^{*}\right\|_{\mathrm{op}}^{s}<1, \\
& \mathbb{E}\left[\mathbf{1}_{\left\{T_{r}^{(t)} \leq \tau\right\}}\left\|A_{r}^{(t)}\right\|_{\mathrm{op}}^{s}\right] \rightarrow 0
\end{aligned}
$$


for every $\tau>0$ and $r=1, \ldots, K$. Then $X_{t}$ converges in distribution to a limit $X$, and

$$
\zeta_{s}\left(X_{t}, X\right) \rightarrow 0, \quad t \rightarrow \infty
$$

where $\mathcal{L}(X)$ is the unique fixed point of $T$ given in (5.6) subject to $\|X\|_{s}<\infty$ and

$$
\begin{cases}\mathbb{E} X=0 & \text { for } 1<s \leq 2, \\ \mathbb{E} X=0, \operatorname{Cov}(X)=\operatorname{Id}_{d} & \text { for } 2<s \leq 3 .\end{cases}
$$

Proof. This proof is a continuous extension of the proof of Theorem 4.1 in Neininger and Rüschendorf 24] for the discrete time case. The existence and uniqueness of the fixed point of $T$ subject to (5.11) is obtained as follows: For $1<s \leq 3$ equation (5.3) implies $\mathbb{E} b^{(t)}=0$ for all $t>0$, thus by (5.7) we obtain $\mathbb{E} b^{*}=0$. For $2<s \leq 3$ equation (5.3) implies that for all $t \geq \tau_{1}$

$$
\begin{aligned}
\operatorname{Id}_{d} & =\operatorname{Cov}\left(X_{t}\right) \\
& =\mathbb{E}\left[b^{(t)}\left(b^{(t)}\right)^{\operatorname{tr}}\right]+\mathbb{E}\left[\sum_{r=1}^{K}\left(\mathbf{1}_{\left\{T_{r}^{(t)}<\tau_{1}\right\}} A_{r}^{(t)} \tilde{C}_{T_{r}^{(t)}}\left(A_{r}^{(t)}\right)^{\operatorname{tr}}+\mathbf{1}_{\left\{T_{r}^{(t)} \geq \tau_{1}\right\}} A_{r}^{(t)}\left(A_{r}^{(t)}\right)^{\operatorname{tr}}\right)\right],
\end{aligned}
$$

where $b^{\text {tr }}$ denotes the transpose of a vector or matrix and $\tilde{C}_{t}:=\operatorname{Cov}\left(X_{t}\right)$; recall that $\tilde{C}_{t}=\mathrm{Id}$ when $t \geq \tau_{1}$.

By (5.7), (5.9) and Hölder's inequality this implies

$$
\mathbb{E}\left[b^{*}\left(b^{*}\right)^{\operatorname{tr}}\right]+\mathbb{E}\left[\sum_{r=1}^{K} A_{r}^{*}\left(A_{r}^{*}\right)^{\operatorname{tr}}\right]=\operatorname{Id}_{d} .
$$

Now, Corollary 3.4 in 24] implies existence and uniqueness of the fixed-point.

Since

$$
\mathbb{E} \sum_{r=1}^{K}\left\|A_{r}^{(t)}\right\|_{\mathrm{op}}^{s} \rightarrow \mathbb{E} \sum_{r=1}^{K}\left\|A_{r}^{*}\right\|_{\mathrm{op}}^{s}=\xi<1
$$

there exist $\xi_{+} \in(\xi, 1)$ and $\tau_{2}>\tau_{1}$ such that for all $t \geq \tau_{2}$ we have

$$
\mathbb{E} \sum_{r=1}^{K}\left\|A_{r}^{(t)}\right\|_{\mathrm{op}}^{s} \leq \xi_{+}<1
$$

Now, we introduce the quantity

$$
Q_{t}:=\sum_{r=1}^{K} A_{r}^{(t)}\left(\mathbf{1}_{\left\{T_{r}^{(t)}<\tau_{2}\right\}} X_{T_{r}^{(t)}}^{(r)}+\mathbf{1}_{\left\{T_{r}^{(t)} \geq \tau_{2}\right\}} X^{(r)}\right)+b^{(t)}, \quad t \geq \tau_{1},
$$

where $\left(A_{1}^{(t)}, \ldots, A_{K}^{(t)}, b^{(t)}, T^{(t)}\right), X^{(1)}, \ldots, X^{(K)},\left(X_{t}^{(1)}\right), \ldots,\left(X_{t}^{(K)}\right)$ are independent with $X^{(r)} \sim X$ and $X_{t}^{(r)} \sim X_{t}$ for $r=1, \ldots, K$ and $t \geq 0$. Comparing with (5.3) we obtain that $Q_{t}$ is centered for $1<s \leq 3$ and has the covariance matrix $\operatorname{Id}_{d}$ for 
$2<s \leq 3$ and $t \geq \tau_{1}$. Hence, $\zeta_{s}$ distances between $X_{t}, Q_{t}$ and $X$ are finite for all $t \geq \tau_{1}$. The triangle inequality implies

$$
\Delta(t):=\zeta_{s}\left(X_{t}, X\right) \leq \zeta_{s}\left(X_{t}, Q_{t}\right)+\zeta_{s}\left(Q_{t}, X\right) .
$$

As in the proof for the discrete case we obtain $\zeta_{s}\left(Q_{t}, X\right) \rightarrow 0$ as $t \rightarrow 0$, where we use that $\sup _{0 \leq t \leq \tau_{2}}\left\|X_{t}\right\|_{s}<\infty$.

The first summand of (5.15) requires a continuous analog of the estimate in the discrete case. Using the properties of the $\zeta_{s}$ metric, we obtain, for $t \geq \tau_{1}$,

$$
\zeta_{s}\left(X_{t}, Q_{t}\right) \leq \mathbb{E} \sum_{r=1}^{K} \mathbf{1}_{\left\{T_{r}^{(t)} \geq \tau_{2}\right\}}\left\|A_{r}^{(t)}\right\|_{\mathrm{op}}^{s} \Delta\left(T_{r}^{(t)}\right),
$$

and, with (5.15), and $r_{t}:=\zeta_{s}\left(Q_{t}, X\right)$ it follows

$$
\Delta(t) \leq \mathbb{E} \sum_{r=1}^{K} \mathbf{1}_{\left\{T_{r}^{(t)} \geq \tau_{2}\right\}}\left\|A_{r}^{(t)}\right\|_{\text {op }}^{s} \Delta\left(T_{r}^{(t)}\right)+r_{t}
$$

Now, we obtain $\Delta(t) \rightarrow 0$ in two steps, first showing that $(\Delta(t))_{t \geq 0}$ is bounded and then, using the bound, that $\Delta(t) \rightarrow 0$.

For the first step we introduce

$$
\Delta^{*}(t):=\sup _{\tau_{2} \leq u \leq t} \Delta(u) .
$$

We have $\Delta^{*}(t)<\infty$ for all $t \geq \tau_{2}$, since, for $\tau_{2} \leq u \leq t$, we have $\zeta_{s}\left(X_{u}, X\right) \leq$ $C_{s}\left(\|X\|_{s}^{s}+\left\|X_{u}\right\|_{s}^{s}\right) \leq C_{s}\left(\|X\|_{s}^{s}+\sup _{\tau_{2} \leq u \leq t}\left\|X_{u}\right\|_{s}^{s}\right)<\infty$ with a constant $C_{s}>0$, using [29, Lemma 2]. By definition, $\Delta^{*}$ is monotonically increasing. With $R:=$ $\sup _{t \geq \tau_{2}} r_{t}<\infty$ we obtain for $\tau_{2} \leq u \leq t$, from (5.17), (5.18) and (5.13),

$$
\begin{aligned}
\Delta(u) & \leq \mathbb{E} \sum_{r=1}^{K} \mathbf{1}_{\left\{T_{r}^{(u)} \geq \tau_{2}\right\}}\left\|A_{r}^{(u)}\right\|_{\mathrm{op}}^{s} \Delta^{*}(u)+R \\
& \leq \xi_{+} \Delta^{*}(t)+R .
\end{aligned}
$$

Hence, we obtain $\Delta^{*}(t) \leq \xi_{+} \Delta^{*}(t)+R$, thus $\Delta^{*}(t) \leq R /\left(1-\xi_{+}\right)$. This implies

$$
\Delta^{*}(\infty):=\sup _{t \geq \tau_{2}} \Delta(t) \leq \frac{R}{1-\xi_{+}}<\infty .
$$

For the second step we denote $L:=\limsup _{t \rightarrow \infty} \Delta(t)$. For every $\varepsilon>0$ there exists a $\tau_{3}>\tau_{2}$ such that we have $\Delta(t) \leq L+\varepsilon$ for all $t \geq \tau_{3}$. Thus, from (5.17) we obtain $\Delta(t) \leq \mathbb{E} \sum_{r=1}^{K} \mathbf{1}_{\left\{\tau_{2} \leq T_{r}^{(t)}<\tau_{3}\right\}}\left\|A_{r}^{(t)}\right\|_{\mathrm{op}}^{s} \Delta^{*}(\infty)+\mathbb{E} \sum_{r=1}^{K} \mathbf{1}_{\left\{T_{r}^{(t)} \geq \tau_{3}\right\}}\left\|A_{r}^{(t)}\right\|_{\mathrm{op}}^{s}(L+\varepsilon)+r_{t}$ and letting $t \rightarrow \infty$ we obtain by (5.9) and (5.12)

$$
L \leq \xi(L+\varepsilon) .
$$

If $L>0$, this is a contradiction for $0<\varepsilon<L(1-\xi) / \xi$. Hence, we have $L=0$. This proves (5.10). Finally, recall that convergence in $\zeta_{s}$ implies weak convergence. 
As a corollary we formulate a univariate central limit theorem that corresponds to Neininger and Rüschendorf [24, Corollary 5.2] for the discrete time case. For this we assume that there are expansions, as $t \rightarrow \infty$,

$$
\mathbb{E} Y_{t}=f(t)+o\left(g^{1 / 2}(t)\right), \quad \operatorname{Var}\left(Y_{t}\right)=g(t)+o(g(t))
$$

with functions $f:[0, \infty) \rightarrow \mathbb{R}, g:[0, \infty) \rightarrow[0, \infty)$, with

$$
\sup _{u \leq t}|f(u)|<\infty \text { for every } t>0, \quad \lim _{t \rightarrow \infty} g(t)=\infty, \quad \sup _{u \leq t} g(u)=O(g(t)) .
$$

Thus, for some constant $C \geq 1, g(u) \leq C g(t)$ when $0 \leq u \leq t$.

Then the following central limit law holds:

Corollary 5.2. Let $2<s \leq 3$ and let $Y_{t}, t \geq 0$, be given $s$-integrable, univariate random variables satisfying (5.1) with $A_{r}(t)=1$ for all $r=1, \ldots, K$ and $t \geq 0$. Assume that $\sup _{u \leq t} \mathbb{E}\left|Y_{u}\right|^{s}<\infty$ for every $t$, and that the mean and variance of $Y_{t}$ satisfy (5.20) with (5.21). If, as $t \rightarrow \infty$,

$$
\begin{aligned}
& \left(\sqrt{\frac{g\left(T_{1}^{(t)}\right)}{g(t)}}, \ldots, \sqrt{\frac{g\left(T_{K}^{(t)}\right)}{g(t)}}\right) \stackrel{\ell_{s}}{\longrightarrow}\left(A_{1}^{*}, \ldots, A_{K}^{*}\right), \\
& \frac{1}{g^{1 / 2}(t)}\left(b_{t}-f(t)+\sum_{r=1}^{K} f\left(T_{r}^{(t)}\right)\right) \stackrel{\ell_{s}}{\longrightarrow} 0,
\end{aligned}
$$

and furthermore

$$
\sum_{r=1}^{K}\left(A_{r}^{*}\right)^{2}=1, \quad \mathbb{P}\left(\bigcup_{r=1}^{K}\left\{A_{r}^{*}=1\right\}\right)<1,
$$

then

$$
\frac{Y_{t}-f(t)}{g^{1 / 2}(t)} \stackrel{\mathrm{d}}{\rightarrow} \mathcal{N}(0,1)
$$

Proof. We begin by replacing $g(t)$ by $\max (g(t), 1)$; by (5.21), this does not affect $g(t)$ for large $t$, and it is easy to see that (5.20), (5.21), (5.22), (5.23) still hold. We may thus assume that $g(t) \geq 1$ for every $t$.

Denote $M_{t}:=\mathbb{E} Y_{t}$ and $\sigma_{t}^{2}:=\operatorname{Var}\left(Y_{t}\right)$. By (5.20), $\sigma_{t}^{2} / g(t) \rightarrow 1$. (All unspecified limits are as $t \rightarrow \infty$.) Choose $\tau_{1} \geq \tau_{0}$ such that $\frac{1}{4} g(t) \leq \sigma_{t}^{2} \leq 4 g(t)$ for $t \geq \tau_{1}$. Let, as in Convention C, $C_{t}:=1$ for $t<\tau_{1}$ and $C_{t}:=\sigma_{t}^{2}$ for $t \geq \tau_{1}$, and write $\tilde{\sigma}_{t}:=C_{t}^{1 / 2}$ and $\varepsilon(t):=\tilde{\sigma}_{t} / g(t)^{1 / 2}-1=\left(C_{t} / g(t)\right)^{1 / 2}-1$. For $t \geq \tau_{1}, \varepsilon(t)=\left(\operatorname{Var} Y_{t} / g(t)\right)^{1 / 2}-1$, so by (5.20),

$$
\varepsilon(t) \rightarrow 0 \quad \text { as } t \rightarrow \infty
$$


Further, $C_{t} / g(t)=1 / g(t) \leq 1$ for $t<\tau_{1}$, while $C_{t} / g(t)=\sigma_{t}^{2} / g(t) \leq 4$ for $t \geq \tau_{1}$. Hence $|\varepsilon(t)| \leq 1$ for all $t$. With (5.4) and $A_{r}(t)=1$ we have, for $t \geq \tau_{1}$,

$$
\begin{array}{r}
A_{r}^{(t)}=\frac{\tilde{\sigma}_{T_{r}^{(t)}}}{\sigma_{t}}=\frac{\left(1+\varepsilon\left(T_{r}^{(t)}\right)\right) g\left(T_{r}^{(t)}\right)^{1 / 2}}{\sigma_{t}}, \\
b^{(t)}=\sigma_{t}^{-1}\left(b_{t}-M_{t}+\sum_{r=1}^{K} M_{T_{r}^{(t)}}\right) .
\end{array}
$$

Since $g\left(T_{r}^{(t)}\right) \leq C g(t)$ by (5.21), we have, for $t \geq \tau_{1}$,

$$
\left\|A_{r}^{(t)}-\frac{g\left(T_{r}^{(t)}\right)^{1 / 2}}{\sigma_{t}}\right\|_{s}=\left\|\varepsilon\left(T_{r}^{(t)}\right) \frac{g\left(T_{r}^{(t)}\right)^{1 / 2}}{\sigma_{t}}\right\|_{s} \leq \sup _{u \leq t}\left|\varepsilon(u) \frac{g(u)^{1 / 2}}{\sigma_{t}}\right| .
$$

For any $\delta>0$, there exists, by (5.26), $\tau(\delta) \geq \tau_{1}$ such that $|\varepsilon(t)| \leq \delta$ when $t \geq \tau(\delta)$. Thus, if $\tau(\delta) \leq u \leq t$, then

$$
\left|\varepsilon(u) \frac{g(u)^{1 / 2}}{\sigma_{t}}\right| \leq \delta \frac{C g(t)^{1 / 2}}{\sigma_{t}} \leq 2 C \delta .
$$

On the other hand, if $u \leq \tau(\delta)$, then

$$
\left|\varepsilon(u) \frac{g(u)^{1 / 2}}{\sigma_{t}}\right| \leq \frac{C g(\tau(\delta))^{1 / 2}}{\sigma_{t}} \rightarrow 0
$$

as $t \rightarrow \infty$. Hence, $\sup _{u \leq t}\left|\varepsilon(u) g(u)^{1 / 2} / \sigma_{t}\right| \leq 2 C \delta$ for sufficiently large $t$. Since $\delta>0$ is arbitrary, it follows that the right hand side of (5.29) tends to 0 as $t \rightarrow \infty$, and thus (5.29) yields

$$
\left\|A_{r}^{(t)}-\frac{g\left(T_{r}^{(t)}\right)^{1 / 2}}{\sigma_{t}}\right\|_{s} \rightarrow 0
$$

Since $g(t)^{1 / 2} / \sigma_{t} \rightarrow 1$, (5.22) yields $g\left(T_{r}^{(t)}\right)^{1 / 2} / \sigma_{t} \stackrel{\ell_{s}}{\longrightarrow} A_{r}^{*}$, which combined with (5.30) yields $A_{r}^{(t)} \stackrel{\ell_{s}}{\longrightarrow} A_{r}^{*}$, jointly for $r=1, \ldots, k$.

Next, for any $\varepsilon>0$, there exists by (5.20) $\tau_{\varepsilon} \geq \tau_{1}$ such that $\left|M_{t}-f(t)\right| \leq \varepsilon g(t)^{1 / 2}$ if $t \geq \tau_{\varepsilon}$. Consequently, if $T_{r}^{(t)} \geq \tau_{\varepsilon}$, then

$$
\left|M_{T_{r}^{(t)}}-f\left(T_{r}^{(t)}\right)\right| \leq \varepsilon g\left(T_{r}^{(t)}\right)^{1 / 2} \leq C \varepsilon g(t)^{1 / 2} .
$$

Since $\sup _{u \leq \tau_{\varepsilon}}\left|M_{u}\right|$ and $\sup _{u \leq \tau_{\varepsilon}}|f(u)|$ are finite, the same estimate holds for $T_{r}^{(t)}<$ $\tau_{\varepsilon}$ too, provided $t$ is large. Consequently, $\left|M_{T_{r}^{(t)}}-f\left(T_{r}^{(t)}\right)\right| / g(t)^{1 / 2} \leq C \varepsilon$ if $t$ is large enough. It follows that $\left\|M_{T_{r}^{(t)}}-f\left(T_{r}^{(t)}\right)\right\|_{s} / g(t)^{1 / 2} \rightarrow 0$ as $t \rightarrow \infty$, so by (5.28),

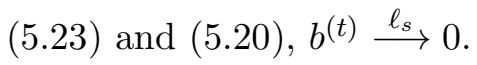

We apply Theorem [5.1] with $2<s \leq 3$; we have shown that (5.7) holds with $b^{*}=$ 0 . The two assumptions in (5.24) and $s>2$ ensure that we have $\mathbb{E} \sum_{r=1}^{K}\left|A_{r}^{*}\right|^{s}<1$. 
Finally, by (5.30), for every $\tau$ and $r$,

$$
\begin{aligned}
\left\|\mathbf{1}_{\left\{T_{r}^{(t)} \leq \tau\right\}} A_{r}^{(t)}\right\|_{s} & \leq\left\|\mathbf{1}_{\left\{T_{r}^{(t)} \leq \tau\right\}} \frac{g\left(T_{r}^{(t)}\right)^{1 / 2}}{\sigma_{t}}\right\|_{s}+\left\|A_{r}^{(t)}-\frac{g\left(T_{r}^{(t)}\right)^{1 / 2}}{\sigma_{t}}\right\|_{s} \\
& \leq \frac{C g(\tau)^{1 / 2}}{\sigma_{t}}+o(1) \rightarrow 0 .
\end{aligned}
$$

Now, Theorem 5.1 implies $\left(Y_{t}-M_{t}\right) / \sigma_{t} \stackrel{\mathrm{d}}{\rightarrow} X$, where $\mathcal{L}(X)$ is characterized by $\|X\|_{s}<\infty, \mathbb{E} X=0, \operatorname{Var}(X)=1$, and

$$
X \stackrel{d}{=} \sum_{r=1}^{K} A_{r}^{*} X^{(r)},
$$

with assumptions as in (5.5). Since $\sum_{r=1}^{K}\left(A_{r}^{*}\right)^{2}=1$ this is solved by $\mathcal{L}(X)=\mathcal{N}(0,1)$. Consequently,

$$
\frac{Y_{t}-M_{t}}{\sigma_{t}} \stackrel{\mathrm{d}}{\rightarrow} \mathcal{N}(0,1)
$$

which, in view of (5.20), implies the assertion.

The following theorem covers cases where the previous central limit theorem of Corollary 5.2 fails due to the appearance of periodic behavior. For this we assume that there is an expansion of the mean, as $t \rightarrow \infty$,

$$
\mathbb{E} Y_{t}=f(t)+\operatorname{Re}\left(\gamma t^{\lambda}\right)+o\left(t^{\sigma}\right)
$$

with a function $f:[0, \infty) \rightarrow \mathbb{R}, \gamma \in \mathbb{C} \backslash\{0\}$, and $\lambda \in \mathbb{C}$ with $\sigma:=\operatorname{Re}(\lambda)>0$. We denote

$$
\begin{aligned}
A_{r}^{(t)} & :=\left(\frac{T_{r}^{(t)}}{t}\right)^{\lambda}, \quad r=1, \ldots, K, \\
b^{(t)} & :=\frac{1}{t^{\sigma}}\left(b_{t}-f(t)+\sum_{r=1}^{K} f\left(T_{r}^{(t)}\right)\right) .
\end{aligned}
$$

Note that $A_{r}^{(t)}$ in general is complex, while $b^{(t)}$ is real.

Theorem 5.3. Let $Y_{t}, t \geq 0$, be given square-integrable, univariate random variables satisfying (5.1) with $A_{r}(t)=1$ for all $r=1, \ldots, K$ and $t \geq 0$. Assume that $\sup _{u \leq t} \mathbb{E}\left|Y_{u}\right|^{2}<\infty$ for every $t>0$ and that the mean of $Y_{t}$ satisfies (5.32) with $\lambda=\sigma+\mathrm{i} \tau$ and $\sigma>0$, and some locally bounded function $f(t)$. If, as $t \rightarrow \infty$,

$$
\left(A_{1}^{(t)}, \ldots, A_{K}^{(t)}\right) \stackrel{\ell_{2}}{\longrightarrow}\left(A_{1}^{*}, \ldots, A_{K}^{*}\right) \quad \text { and } \quad\left\|b^{(t)}\right\|_{2} \rightarrow 0,
$$

and furthermore

$$
\mathbb{E} \sum_{r=1}^{K}\left|A_{r}^{*}\right|^{2}<1
$$


then, as $t \rightarrow \infty$,

$$
\ell_{2}\left(\frac{Y_{t}-f(t)}{t^{\sigma}}, \operatorname{Re}\left(X e^{\mathrm{i} \tau \ln t}\right)\right) \rightarrow 0
$$

where $\mathcal{L}(X)$ is the unique fixed point in $\mathcal{M}_{2}^{\mathbb{C}}(\gamma)$ of

$$
T: \mathcal{M}^{\mathbb{C}} \rightarrow \mathcal{M}^{\mathbb{C}}, \quad \eta \mapsto \mathcal{L}\left(\sum_{r=1}^{K} A_{r}^{*} Z^{(r)}\right)
$$

where $\left(A_{1}^{*}, \ldots, A_{K}^{*}\right), Z^{(1)}, \ldots, Z^{(K)}$ are independent and $\mathcal{L}\left(Z^{(r)}\right)=\eta$ for $r=$ $1, \ldots, K$.

Proof. We extend an approach based on the contraction method from Fill and Kapur 11]. We may assume that $\tau_{0} \geq 1$.

First, for technical convenience we show that we further may assume $Y_{t}=0$ and $f(t)=0$ for $0 \leq t \leq 1$. Let $\left(Y_{t}^{(r) *}\right)_{t}, r=1, \ldots, K$, be another set of copies of $\left(Y_{t}\right)_{t}$, independent of each other and of everything else. We may replace $Y_{t}^{(r)}$ in (5.1) by $Y_{t}^{(r)} \mathbf{1}_{\{t \geq 1\}}+Y_{t}^{(r) *} \mathbf{1}_{\{t<1\}}$, which has the same distribution and independence properties. Hence $Y_{t}^{(r)} \mathbf{1}_{\{t \geq 1\}}$ satisfies (5.1) (for $t \geq \tau_{0} \geq 1$ ) with $b_{t}$ replaced by $\tilde{b}_{t}:=b_{t}+\sum_{r} Y_{T_{r}^{(t)}}^{(r) *} \mathbf{1}_{\left\{T_{r}^{(t)}<1\right\}}$. This replaces $b^{(t)}$ by $\tilde{b}^{(t)}$ with

$$
\left|\tilde{b}^{(t)}-b^{(t)}\right| \leq t^{-\sigma} \sum_{r}\left|Y_{T_{r}^{(t)}}^{(r) *}\right| \mathbf{1}_{\left\{T_{r}^{(t)}<1\right\}}
$$

so $\left\|\tilde{b}^{(t)}-b^{(t)}\right\|_{2}=O\left(t^{-\sigma}\right)$ and (5.35) still holds. We may thus consider $Y_{t}^{(r)} \mathbf{1}_{\{t \geq 1\}}$ instead, and thus we may assume that $Y_{t}^{(r)}=0$ when $t<1$. Similarly, we may assume that $f(t)=0$ for $t<1$, changing $b^{(t)}$ by $O\left(t^{-\sigma}\right)$.

With $X_{t}:=\left(Y_{t}-f(t)\right) / t^{\sigma}$ for $t>0$ and $X_{0}:=0$ we obtain

$$
X_{t} \stackrel{\mathrm{d}}{=} \sum_{r=1}^{K}\left(\frac{T_{r}^{(t)}}{t}\right)^{\sigma} X_{T_{r}^{(t)}}^{(r)}+b^{(t)}, \quad t \geq \tau_{0},
$$

with $b^{(t)}$ as given in (5.34).

Next we prove that the restriction of $T$ defined in (5.38) to $\mathcal{M}_{2}^{\mathbb{C}}(\gamma)$ maps into $\mathcal{M}_{2}^{\mathbb{C}}(\gamma)$ and is Lipschitz in $\ell_{2}$ with Lipschitz constant bounded by $\left(\mathbb{E} \sum_{r=1}^{K}\left|A_{r}^{*}\right|^{2}\right)^{1 / 2}<1$.

Note that (5.36) implies $\left\|A_{r}^{*}\right\|_{2}<\infty$ for all $r=1, \ldots, K$. This implies that $T(\eta)$ has a finite second moment for all $\eta \in \mathcal{M}_{2}^{\mathbb{C}}$. Next we claim that $\sum_{r=1}^{K} \mathbb{E} A_{r}^{*}=1$. This implies that $T(\eta)$ has mean $\gamma$ for all $\eta \in \mathcal{M}_{2}^{\mathbb{C}}(\gamma)$. To prove $\sum_{r=1}^{K} \mathbb{E} A_{r}^{*}=1$, note that (5.32) implies $\mathbb{E} X_{t}=\operatorname{Re}\left(\gamma t^{\mathrm{i} \tau}\right)+o(1)$ as $t \rightarrow \infty$. On the other hand, the 
right hand side of (5.39) has mean, using $\mathbb{E} b^{(t)} \rightarrow 0$,

$$
\begin{aligned}
\sum_{r=1}^{K} \mathbb{E}\left[\left(\frac{T_{r}^{(t)}}{t}\right)^{\sigma} \operatorname{Re}\left(\gamma\left(T_{r}^{(t)}\right)^{\mathrm{i} \tau}\right)\right]+o(1) & =\operatorname{Re}\left(\gamma \sum_{r=1}^{K} \mathbb{E} \frac{\left(T_{r}^{(t)}\right)^{\lambda}}{t^{\sigma}}\right)+o(1) \\
& =\operatorname{Re}\left(\gamma t^{\mathrm{i} \tau} \sum_{r=1}^{K} \mathbb{E}\left(\frac{T_{r}^{(t)}}{t}\right)^{\lambda}\right)+o(1) \\
& =\operatorname{Re}\left(\gamma t^{\mathrm{i} \tau} \sum_{r=1}^{K} \mathbb{E} A_{r}^{*}\right)+o(1),
\end{aligned}
$$

where we also used that $\mathbb{E}\left(T_{r}^{(t)} / t\right)^{\lambda} \rightarrow \mathbb{E} A_{r}^{*}$, see (5.35). Hence, together we obtain, as $t \rightarrow \infty$,

$$
\operatorname{Re}\left(\gamma t^{\mathrm{i} \tau}\right)+o(1)=\operatorname{Re}\left(\gamma t^{\mathrm{i} \tau} \sum_{r=1}^{K} \mathbb{E} A_{r}^{*}\right)+o(1) .
$$

Thus, $\gamma \neq 0$ yields $\sum_{r=1}^{K} \mathbb{E} A_{r}^{*}=1$. For the bound on the Lipschitz constant in $\ell_{2}$ of $T$ restricted to $\mathcal{M}_{2}^{\mathbb{C}}$ see Rösler and Rüschendorf [27, Lemma 1] and Fill and Kapur 11]: For $\mu, \nu \in \mathcal{M}_{2}^{\mathbb{C}}$ choose $\left(Z^{(1)}, W^{(1)}\right), \ldots,\left(Z^{(K)}, W^{(K)}\right)$ as identically distributed vectors of optimal couplings of $\mu$ and $\nu$ and such that $\left(Z^{(1)}, W^{(1)}\right), \ldots,\left(Z^{(K)}, W^{(K)}\right)$, $\left(A_{1}^{*}, \ldots, A_{K}^{*}\right)$ are independent. Then we have

$$
\begin{aligned}
\ell_{2}^{2}(T(\mu), T(\nu)) & =\ell_{2}^{2}\left(\sum_{r=1}^{K} A_{r}^{*} Z^{(r)}, \sum_{r=1}^{K} A_{r}^{*} W^{(r)}\right) \\
& \leq \mathbb{E}\left|\sum_{r=1}^{K} A_{r}^{*}\left(Z^{(r)}-W^{(r)}\right)\right|^{2} \\
& =\mathbb{E}\left(\sum_{r=1}^{K}\left|A_{r}^{*}\right|^{2}\left|Z^{(r)}-W^{(r)}\right|^{2}+\sum_{r \neq s} A_{r}^{*}\left(Z^{(r)}-W^{(r)}\right) \overline{A_{s}^{*}\left(Z^{(s)}-W^{(s)}\right)}\right) \\
& =\mathbb{E} \sum_{r=1}^{K}\left|A_{r}^{*}\right|^{2} \ell_{2}^{2}(\mu, \nu)+0 \\
& =\sum_{r=1}^{K} \mathbb{E}\left|A_{r}^{*}\right|^{2} \ell_{2}^{2}(\mu, \nu) .
\end{aligned}
$$

Altogether we obtain that $T$ has a unique fixed point $\mathcal{L}(X)$ in $\mathcal{M}_{2}^{\mathbb{C}}(\gamma)$.

The fixed point property of $\mathcal{L}(X)$ implies

$$
\frac{1}{t^{\sigma}} \operatorname{Re}\left(t^{\lambda} X\right) \stackrel{\mathrm{d}}{=} \frac{1}{t^{\sigma}} \operatorname{Re}\left(\sum_{r=1}^{K} t^{\lambda} A_{r}^{*} X^{(r)}\right) .
$$

where $\left(A_{1}^{*}, \ldots, A_{K}^{*}\right), X^{(1)}, \ldots, X^{(b)}$ are independent and $\mathcal{L}\left(X^{(r)}\right)=\mathcal{L}(X)$ for $r=$ $1, \ldots, K$. We may assume, e.g. by taking optimal couplings, that $\left\|A_{r}^{(t)}-A_{r}^{*}\right\|_{2} \rightarrow 0$ 
as $t \rightarrow \infty$. We choose $X_{t}^{(r)}$ as optimal couplings to $\operatorname{Re}\left(t^{\mathrm{i} \tau} X^{(r)}\right)$ (with the right distribution, i.e. the distribution of $X_{t}$ ) for $t \geq 0$ and $r=1, \ldots, K$. Clearly, we may assume that, as required, $X_{t}^{(r)}, r=1, \ldots, K$, are independent of each other and of $\left(T^{(t)}, b_{t}\right)_{t}$.

We denote, for $t>0$,

$$
\Delta(t):=\ell_{2}\left(\frac{Y_{t}-f(t)}{t^{\sigma}}, \operatorname{Re}\left(X e^{\mathrm{i} \tau \ln t}\right)\right)=\ell_{2}\left(X_{t}, \frac{1}{t^{\sigma}} \operatorname{Re}\left(t^{\lambda} X\right)\right) .
$$

Using (5.39) and (5.41) we obtain, for $t \geq \tau_{0}$,

$$
\begin{aligned}
\Delta(t)= & \ell_{2}\left(\sum_{r=1}^{K}\left(\frac{T_{r}^{(t)}}{t}\right)^{\sigma} X_{T_{r}^{(t)}}^{(r)}+b^{(t)}, \frac{1}{t^{\sigma}} \operatorname{Re}\left(\sum_{r=1}^{K} t^{\lambda} A_{r}^{*} X^{(r)}\right)\right) \\
\leq & \left\|\sum_{r=1}^{K}\left(\left(\frac{T_{r}^{(t)}}{t}\right)^{\sigma} X_{T_{r}^{(t)}}^{(r)}-\frac{1}{t^{\sigma}} \operatorname{Re}\left(t^{\lambda} A_{r}^{*} X^{(r)}\right)\right)\right\|_{2}+\left\|b^{(t)}\right\|_{2} \\
\leq & \left\|\sum_{r=1}^{K}\left(\left(\frac{T_{r}^{(t)}}{t}\right)^{\sigma} X_{T_{r}^{(t)}}^{(r)}-\frac{1}{t^{\sigma}} \operatorname{Re}\left(\left(T_{r}^{(t)}\right)^{\lambda} X^{(r)}\right)\right)\right\|_{2}+\left\|b^{(t)}\right\|_{2} \\
& +\left\|\sum_{r=1}^{K}\left(\frac{1}{t^{\sigma}} \operatorname{Re}\left(\left(T_{r}^{(t)}\right)^{\lambda} X^{(r)}\right)-\frac{1}{t^{\sigma}} \operatorname{Re}\left(t^{\lambda} A_{r}^{*} X^{(r)}\right)\right)\right\|_{2} .
\end{aligned}
$$

By (5.35) and (5.33) the second and third of the three latter summands tend to zero as $t \rightarrow \infty$. We abbreviate

$$
W_{r}^{(t)}:=\left(\frac{T_{r}^{(t)}}{t}\right)^{\sigma} X_{T_{r}^{(t)}}^{(r)}-\frac{1}{t^{\sigma}} \operatorname{Re}\left(\left(T_{r}^{(t)}\right)^{\lambda} X^{(r)}\right) .
$$

Hence, (5.42) implies

$$
\begin{aligned}
\Delta(t) & \leq\left(\mathbb{E}\left(\sum_{r=1}^{K} W_{r}^{(t)}\right)^{2}\right)^{1 / 2}+o(1) \\
& =\left(\mathbb{E} \sum_{r=1}^{K}\left(W_{r}^{(t)}\right)^{2}+\mathbb{E} \sum_{\substack{r, s=1 \\
r \neq s}}^{K} W_{r}^{(t)} W_{s}^{(t)}\right)^{1 / 2}+o(1) .
\end{aligned}
$$

By the definition of $\Delta(t)$ and the fact that $\left(X_{t}^{(r)}, \operatorname{Re}\left(t^{\mathrm{i} \tau} X^{(r)}\right)\right)$ are optimal couplings for all $t>0$ and $r=1, \ldots, K$ we obtain

$$
\mathbb{E}\left(W_{r}^{(t)}\right)^{2}=\mathbb{E}\left[\left(\frac{T_{r}^{(t)}}{t}\right)^{2 \sigma} \Delta^{2}\left(T_{r}^{(t)}\right)\right] .
$$

From (5.32) we obtain

$$
\mathbb{E} X_{t}=\frac{1}{t^{\sigma}} \operatorname{Re}\left(\gamma t^{\lambda}\right)+R(t), \quad t>0
$$


with $R(t) \rightarrow 0$ as $t \rightarrow \infty$. Since $\mathbb{E} X^{(r)}=\gamma$ and by the independence conditions we obtain $\mathbb{E} W_{r}^{(t)}=\mathbb{E}\left[\left(T_{r}^{(t)} / t\right)^{\sigma} R\left(T_{r}^{(t)}\right)\right]$ and, for $r \neq s$,

$$
\mathbb{E}\left[W_{r}^{(t)} W_{s}^{(t)}\right]=\mathbb{E}\left[\left(\frac{T_{r}^{(t)}}{t} \frac{T_{s}^{(t)}}{t}\right)^{\sigma} R\left(T_{r}^{(t)}\right) R\left(T_{s}^{(t)}\right)\right] .
$$

Splitting the latter integral into the events $\left\{T_{r}^{(t)} \leq t_{1}\right.$ or $\left.T_{s}^{(t)} \leq t_{1}\right\}$ and $\left\{T_{r}^{(t)}>\right.$ $t_{1}$ and $\left.T_{s}^{(t)}>t_{1}\right\}$ for some $t_{1}>0$ we obtain, for every $t_{1}>0$,

$$
\left|\mathbb{E}\left[W_{r}^{(t)} W_{s}^{(t)}\right]\right| \leq\left(\frac{t_{1}}{t}\right)^{\sigma}\|R\|_{\infty}^{2}+\sup _{u \geq t_{1}} R^{2}(u)
$$

where $\|R\|_{\infty}:=\sup _{t}|R(t)|<\infty$. From this we obtain first, letting $t \rightarrow \infty$, $\lim \sup _{t \rightarrow \infty}\left|\mathbb{E}\left[W_{r}^{(t)} W_{s}^{(t)}\right]\right| \leq \sup _{u \geq t_{1}} R^{2}(u)$, and then, letting $t_{1} \rightarrow \infty$,

$$
\mathbb{E}\left[W_{r}^{(t)} W_{s}^{(t)}\right] \rightarrow 0 \quad \text { as } t \rightarrow \infty .
$$

Now, (5.44), (5.45), and (5.46) imply, for $t>\tau_{0}$,

$$
\Delta(t) \leq\left(\mathbb{E}\left[\sum_{r=1}^{K}\left(\frac{T_{r}^{(t)}}{t}\right)^{2 \sigma} \Delta^{2}\left(T_{r}^{(t)}\right)\right]+R_{1}(t)\right)^{1 / 2}+R_{2}(t),
$$

with $R_{1}(t), R_{2}(t) \rightarrow 0$ as $t \rightarrow \infty$.

We first show that $\|\Delta\|_{\infty}<\infty$. Define $\Delta^{*}(t):=\sup _{0<u \leq t} \Delta(u)$. By the assumptions $\sup _{0 \leq u \leq t} \mathbb{E}\left|Y_{u}\right|^{2}<\infty$ and $\sup _{0 \leq u \leq t}|f(u)|<\infty$, together with $Y_{u}=0$ and $f(u)=0$ for $u \leq 1$, we have $\Delta^{*}(t)<\infty$ for all $t>0$. Let $t_{1} \geq \tau_{0}$ be such that $\left|R_{1}(t)\right|<1$ and $\left|R_{2}(t)\right|<1$ for $t \geq t_{1}$. Then with (5.47) we obtain, for $t \geq t_{1}$,

$$
\Delta(t) \leq\left(\mathbb{E}\left[\sum_{r=1}^{K}\left(\frac{T_{r}^{(t)}}{t}\right)^{2 \sigma}\left(\Delta^{*}\right)^{2}(t)\right]+1\right)^{1 / 2}+1 .
$$

By (5.33), (5.35) and (5.36) there exists a $t_{2} \geq t_{1}$ such that for all $t \geq t_{2}$ we have $\mathbb{E} \sum_{r=1}^{K}\left(T_{r}^{(t)} / t\right)^{2 \sigma} \leq \xi<1$. Thus, for all $t \geq t_{2}$ we obtain, with $\sqrt{a+b} \leq \sqrt{a}+\sqrt{b}$ for $a, b \geq 0$,

$$
\Delta(t) \leq \sqrt{\xi} \Delta^{*}(t)+2
$$

and thus

$$
\Delta^{*}(t) \leq \sqrt{\xi} \Delta^{*}(t)+2+\Delta^{*}\left(t_{2}\right)
$$

which implies $\|\Delta\|_{\infty} \leq\left(2+\Delta^{*}\left(t_{2}\right)\right) /(1-\sqrt{\xi})<\infty$.

In a second step we show that $\Delta(t) \rightarrow 0$ as $t \rightarrow \infty$. For this we assume that $L:=\limsup _{t \rightarrow \infty} \Delta(t)>0$. Let $\varepsilon>0$. There exists a $t_{3} \geq t_{2}$ such that for all $t \geq t_{3}$ 
we have $\Delta(t) \leq L+\varepsilon$. Then (5.47) implies

$\Delta(t)$

$$
\begin{aligned}
& \leq\left(\mathbb{E}\left[\sum_{r=1}^{K}\left(\frac{T_{r}^{(t)}}{t}\right)^{2 \sigma}\left(\mathbf{1}_{\left\{T_{r}^{(t)}<t_{3}\right\}}+\mathbf{1}_{\left\{T_{r}^{(t)} \geq t_{3}\right\}}\right) \Delta^{2}\left(T_{r}^{(t)}\right)\right]+R_{1}(t)\right)^{1 / 2}+R_{2}(t) \\
& \leq\left(\sum_{r=1}^{K}\left(\frac{t_{3}}{t}\right)^{2 \sigma}\|\Delta\|_{\infty}^{2}+\xi(L+\varepsilon)^{2}+R_{1}(t)\right)^{1 / 2}+R_{2}(t) .
\end{aligned}
$$

Hence, $t \rightarrow \infty$ implies

$$
L \leq \sqrt{\xi}(L+\varepsilon)
$$

which if $L>0$ is a contradiction if we choose $\varepsilon$ small enough. Consequently, we have $L=0$ yielding the assertion.

\section{Proof of Theorem 1.3}

In this section we prove Theorem 1.3. The statements on mean and variance of $N(x)$ are proved in Section 3. It remains to identify the asymptotic distribution of $N(x)$ Note that recurrence (1.3) for $N(x)$ is covered by the general recurrence for $Y_{t}$ in (5.1) by making the choices $d=1, K=b, \tau_{0}=1, A_{r}(t)=1, T_{r}^{(t)}=V_{r} t$ and $b_{t}=1$ for all $r=1, \ldots, K$ and $t \geq \tau_{0}$.

We consider the three cases (i) - (iii) appearing in Theorem 1.3 separately:

Case (i): We have $\mathbb{E} N(x)=\alpha^{-1} x+o(\sqrt{x})$ and $\operatorname{Var}(N(x)) \sim \beta x$ with $\beta>0$. We apply Corollary 5.2 with the choices $f(t)=\alpha^{-1} t$ and $g(t)=\beta t$. The conditions (5.20) and (5.21) are satisfied. We have $\sup _{u \leq t} \mathbb{E}\left|Y_{u}\right|^{s}<\infty$ for $s=3$ by Lemma 3.5. Condition (5.22) is satisfied with $A_{r}^{*}=\sqrt{V_{r}}$ for $r=1, \ldots, K$, condition (5.23) is trivially satisfied, and we have (5.24). Hence, Corollary 5.2 applies and yields

$$
\frac{N(x)-\alpha^{-1} x}{\sqrt{\beta x}} \stackrel{\mathrm{d}}{\rightarrow} \mathcal{N}(0,1)
$$

which is the assertion.

Case (ii): We have $\mathbb{E} N(x)=\alpha^{-1} x+O(\sqrt{x})$ and $\operatorname{Var}(N(x)) \sim \beta x \ln x$ with $\beta>0$. We apply Corollary 5.2 with the choices $f(t)=\alpha^{-1} t$ and $g(t)=\beta t \ln t$. Now we have $g\left(T_{r}^{(t)}\right) / g(t)=V_{r}+V_{r} \ln \left(V_{r}\right) / \ln t$, hence we obtain, since $x \mapsto x \ln x$ is bounded on $[0,1]$,

$$
\left(\sqrt{\frac{g\left(T_{1}^{(t)}\right)}{g(t)}}, \ldots, \sqrt{\frac{g\left(T_{K}^{(t)}\right)}{g(t)}}\right) \quad \stackrel{\ell_{3}}{\longrightarrow}\left(A_{1}^{*}, \ldots, A_{K}^{*}\right)
$$


with $A_{r}^{*}=\sqrt{V_{r}}$ for $r=1, \ldots, K$. All conditions of Corollary 5.2 are satisfied as in case (i) and we obtain

$$
\frac{N(x)-\alpha^{-1} x}{\sqrt{\beta x \ln x}} \stackrel{\mathrm{d}}{\rightarrow} \mathcal{N}(0,1) .
$$

Case (iii): Theorem 5.3 can be applied and yields

$$
\ell_{2}\left(\frac{N(x)-\alpha^{-1} x}{x^{\operatorname{Re} \lambda_{2}}}, \operatorname{Re}\left(\Xi e^{\mathrm{i} \operatorname{Im} \lambda_{2} \ln x}\right)\right) \rightarrow 0
$$

as $x \rightarrow \infty$.

Here, we give a simplified version of the proof of Theorem 5.3 for the special recurrence (1.3) which yields also the rate of convergence stated in Theorem 1.3.

The restriction of $T$ defined in (1.7) to $\mathcal{M}_{2}^{\mathbb{C}}(\nu)$ is Lipschitz in $\ell_{2}$ with Lipschitz constant bounded by $\left(\mathbb{E} \sum_{r=1}^{b} V_{r}^{2 \operatorname{Re}\left(\lambda_{2}\right)}\right)^{1 / 2}$; cf. the first part of the proof of Theorem 5.3 Recall $\lambda_{2}=\sigma+\mathrm{i} \tau$ with real $\sigma, \tau$. Hence, $\sigma>1 / 2$ implies that $T$ has a unique fixed point $\mathcal{L}(\Xi)$ in $\mathcal{M}_{2}^{\mathbb{C}}(\nu)$.

For $X_{t}:=N(t)-\alpha^{-1} t$ we obtain with

$$
X_{t} \stackrel{d}{=} \sum_{r=1}^{b} X_{V_{r} t}^{(r)}+1
$$

where $X_{t}^{(r)}$ are independent distributional copies of $X_{t}$ also independent of $\left(V_{1}, \ldots, V_{b}\right)$. With the fixed point property of $\Xi$ we have

$$
t^{\sigma} \operatorname{Re}\left(\Xi e^{\mathrm{i} \tau \ln x}\right)=\operatorname{Re}\left(t^{\lambda_{2}} \Xi\right) \stackrel{d}{=} \operatorname{Re}\left(\sum_{r=1}^{b}\left(V_{r} t\right)^{\lambda_{2}} \Xi^{(r)}\right),
$$

where $\left(V_{1}, \ldots, V_{b}\right), \Xi^{(1)}, \ldots, \Xi^{(b)}$ are independent and $\mathcal{L}\left(\Xi^{(r)}\right)=\mathcal{L}(\Xi)$ for $r=$ $1, \ldots, b$. We choose $X_{t}^{(r)}$ as optimal couplings to $\operatorname{Re}\left(t^{\lambda_{2}} \Xi^{(r)}\right)$ for $t \geq 0$ and $r=1, \ldots, b$ and denote $\Delta(t):=\ell_{2}\left(X_{t}, \operatorname{Re}\left(t^{\lambda_{2}} \Xi\right)\right)$. Note that in the definition of $X_{t}$ we did not rescale by $t^{\sigma}$, hence we have to show $\Delta(t)=O\left(t^{\kappa}\right)$.

With $W_{r}^{(t)}:=X_{V_{r} t}^{(r)}-\operatorname{Re}\left(\left(V_{r} t\right)^{\lambda_{2}} \Xi^{(r)}\right)$ we obtain, for $t \geq 1$,

$$
\begin{aligned}
\Delta(t) & =\ell_{2}\left(\sum_{r=1}^{b} X_{V_{r} t}^{(r)}+1, \sum_{r=1}^{b} \operatorname{Re}\left(\left(V_{r} t\right)^{\lambda_{2}} \Xi^{(r)}\right)\right) \\
& \leq\left\{\mathbb{E}\left(\sum_{r=1}^{b} W_{r}^{(t)}\right)^{2}\right\}^{1 / 2}+1 \\
& =\left\{\sum_{r=1}^{b} \mathbb{E}\left(W_{r}^{(t)}\right)^{2}+\sum_{\substack{r, s=1 \\
r \neq s}}^{b} \mathbb{E}\left[W_{r}^{(t)} W_{s}^{(t)}\right]\right\}^{1 / 2}+1 .
\end{aligned}
$$

Conditioning on $\left(V_{1}, \ldots, V_{b}\right)$ yields $\mathbb{E}\left(W_{r}^{(t)}\right)^{2}=\mathbb{E} \Delta^{2}\left(V_{r} t\right)$. From $\mathbb{E} N(t)=\alpha^{-1} t+$ $\operatorname{Re}\left(\gamma t^{\lambda_{2}}\right)+O\left(t^{\kappa}\right)$ and $\mathbb{E} \Xi=\gamma$ we obtain $\mathbb{E} W_{r}^{(t)}=O\left(t^{\kappa}\right)$. Since $W_{r}^{(t)}$ and $W_{s}^{(t)}$ are 
independent for $r \neq s$ conditionally on $\left(V_{1}, \ldots, V_{b}\right)$, it follows that

$$
\Delta(t) \leq\left\{\sum_{r=1}^{b} \mathbb{E} \Delta^{2}\left(V_{r} t\right)+O\left(t^{2 \kappa}\right)\right\}^{1 / 2}+1, \quad t \geq 1
$$

Now, we show that $\Delta(t) / t^{\kappa}=O(1)$. Note that this implies the assertion. We denote

$$
\Psi^{*}(t):=\sup _{1 \leq u \leq t} \frac{\Delta(u)}{u^{\kappa}}
$$

Then, (6.2) implies, that for appropriate $R>0$

$$
\Psi^{*}(t) \leq\left\{\sum_{r=1}^{b} \mathbb{E} V_{r}^{2 \kappa}\left(\Psi^{*}\right)^{2}(t)+R\right\}^{1 / 2}+1, \quad t \geq 1
$$

and, with $\sqrt{a+b} \leq \sqrt{a}+\sqrt{b}$ for $a, b \geq 0$ and $\xi=\mathbb{E} \sum_{r=1}^{b} V_{r}^{2 \kappa}<1$ this implies

$$
\Psi^{*}(t) \leq \frac{\sqrt{R}+1}{1-\sqrt{\xi}}<\infty
$$

The assertion follows.

\section{EXAMPLES}

Example 7.1 (Random splitting of intervals). Sibuya and Itoh [28] studied the case of random splitting of intervals, with uniformly distributed splitting points; this is the case $b=2$ and $\mathbf{V}=(U, 1-U)$, with $U \sim \mathrm{U}(0,1)$.

We have

$$
\phi(z)=\mathbb{E} U^{z}+\mathbb{E}(1-U)^{z}=2 \int_{0}^{1} u^{z} d u=\frac{2}{1+z}, \quad \operatorname{Re} z>-1
$$

which is a rational function. The characteristic equation (1.5) is $2 /(1+\lambda)=1$, and has the single root $\lambda=1$. Thus Theorem 1.3(i) applies and shows asymptotic normality, as stated by Dean and Majumdar [7. Further, $\alpha=-\phi^{\prime}(1)=1 / 2$, so Theorem 3.1(ii) yields $\mathbb{E} N(x)=m(x)=2 x+O\left(x^{\delta}\right)$ for every $\delta>0$. More precisely, Theorem 3.4 yields

$$
\mathbb{E} N(x)=m(x)=2 x-1, \quad x \geq 1,
$$

which also can be shown directly from (1.2) or from (3.11). 
For the asymptotic variance, we obtain from Theorem [3.4(ii), since $M=1$ and $a_{0}=-1$, using symmetry,

$$
\begin{aligned}
\beta= & \alpha^{-1}(\mathbb{E}(U+2 U \wedge(1-U)+1-U)-2+1) \\
& +2 \alpha^{-2}\left(2 \mathbb{E}\left(U(\ln (1-U)-\ln U) \mathbf{1}_{\{U<1-U\}}\right)\right)-2 \alpha^{-1} \\
& +\alpha^{-3}(2 \mathbb{E}(U \wedge(1-U)))-\alpha^{-1} \\
= & 20 \mathbb{E}(U \wedge(1-U))+16 \int_{0}^{1 / 2} u(\ln (1-u)-\ln (u)) d u-6 \\
= & 8 \ln 2-5 \approx 0.545177 .
\end{aligned}
$$

This can also be obtained from Theorem 3.1(iii); we have

$$
\begin{aligned}
\psi(z, w) & =\mathbb{E}\left(\left(U^{z}+(1-U)^{z}\right)\left(U^{w}+(1-U)^{w}\right)\right)-\phi(z) \phi(w) \\
& =\frac{2}{1+z+w}+2 B(z+1, w+1)-\frac{4}{(1+z)(1+w)} \\
& =\frac{2}{1+z+w}+2 \frac{\Gamma(z+1) \Gamma(w+1)}{\Gamma(z+w+2)}-\frac{4}{(1+z)(1+w)}
\end{aligned}
$$

and thus

$$
\begin{aligned}
\psi(1 / 2+\mathrm{i} u, 1 / 2-\mathrm{i} u) & =1+\Gamma(3 / 2+\mathrm{i} u) \Gamma(3 / 2-\mathrm{i} u)-\frac{4}{|3 / 2+\mathrm{i} u|^{2}} \\
& =1+|1 / 2+\mathrm{i} u|^{2} \frac{\pi}{\cosh \pi u}-\frac{4}{|3 / 2+\mathrm{i} u|^{2}},
\end{aligned}
$$

and, since $1-\phi(z)=(z-1) /(z+1)$,

$$
\beta=\frac{1}{\pi} \int_{-\infty}^{\infty}\left(1+\frac{\pi}{\cosh \pi u}|1 / 2+\mathrm{i} u|^{2}-\frac{4}{|3 / 2+\mathrm{i} u|^{2}}\right) \frac{|3 / 2+\mathrm{i} u|^{2}}{|1 / 2+\mathrm{i} u|^{4}} d u
$$

which can be integrated (with some effort) to yield $8 \ln 2-5$.

Consequently, by Theorem 1.3 .

$$
\frac{N(x)-2 x}{\sqrt{x}} \stackrel{\mathrm{d}}{\rightarrow} \mathcal{N}(0,8 \ln 2-5) .
$$

Example 7.2 ( $m$-ary splitting of intervals). We can generalize Example 7.1 by splitting each interval into $m$ parts, where $m \geq 2$ is fixed, using $m-1$ independent, uniformly distributed cut points in each interval. This has been studied by Dean and Majumdar [7].

We have $b=m$, and $V_{1}, \ldots, V_{m}$ have the same distribution with density ( $m-$ 1) $(1-x)^{b-2}, 0<x<1$. Hence,

$$
\begin{aligned}
\phi(z) & =m \mathbb{E} V_{1}^{z}=m(m-1) \int_{0}^{1} x^{z}(1-x)^{z} d x=m(m-1) B(z+1, m-1) \\
& =\frac{\Gamma(z+1) m !}{\Gamma(m+z)}=\frac{m !}{(z+1) \cdots(z+m-1)} .
\end{aligned}
$$


The characteristic equation $\phi(z)=1$ becomes $\Gamma(z+m) / \Gamma(z+1)=m$ !, or

$$
(z+1) \cdots(z+m-1)=m !
$$

The same equation appears in the analysis of $m$-ary search trees. It is shown by Mahmoud and Pittel [22] and Fill and Kapur [12] that if $m \leq 26$, then $\operatorname{Re} \lambda_{2}<1 / 2$, and thus (i) applies, but if $m \geq 27$, then $\operatorname{Re} \lambda_{2}>1 / 2$, see also, e.g., Chauvin and Pouyanne [3] and Chern and Hwang [5]. Theorem 3.4 yields an exact formula for $\mathbb{E} N(x)$ (although it is hardly useful except when $m$ is small). It further leads to a formula for the asymptotic variance, provided $m \leq 26$.

We have, with $\psi(z):=\Gamma^{\prime}(z) / \Gamma(z)$ and $H_{z}:=\psi(z+1)-\psi(1)$ (for integer $z$, these are the harmonic numbers)

$$
\alpha=-\phi^{\prime}(1)=\psi(m+1)-\psi(2)=H_{m}-1 .
$$

Example 7.3 (Random splitting of multidimensional intervals). Another generalization is to consider $d$-dimensional intervals, where an interval is split into $2^{d}$ subintervals by $d$ hyperplanes orthogonal to the coordinate axis and passing through a random, uniformly distributed point. This too has been studied by Dean and Majumdar 7 .

We have $b=2^{d}$. $V_{1}, \ldots, V_{b}$ have the same distribution, $V_{j} \stackrel{\mathrm{d}}{=} U_{1} \cdots U_{d}$, where $U_{k} \sim U(0,1)$ are i.i.d. Hence,

$$
\phi(z)=2^{d} \mathbb{E} V_{1}^{z}=2^{d}\left(\mathbb{E} U_{1}^{z}\right)^{d}=\left(\frac{2}{1+z}\right)^{d} .
$$

Again, $\phi$ is rational. The characteristic equation may be written $((1+\lambda) / 2)^{d}=1$, with the roots

$$
\Lambda=\left\{2 e^{2 \pi \mathrm{i} k / d}-1: 0 \leq k \leq d-1\right\} .
$$

Thus $\sigma_{2}:=\operatorname{Re} \lambda_{2}=2 \cos \frac{2 \pi}{d}-1$, and the condition $\operatorname{Re} \lambda_{2}<1 / 2$ is equivalent to $\cos (2 \pi / d)<3 / 4$, which holds for $d \leq 8$, while $\operatorname{Re} \lambda_{2}>1 / 2$ for $d \geq 9$. This justifies the claims in Dean and Majumdar [7].

The same characteristic equation, and the same phase transition, appears for quad trees, see Chern, Fuchs and Hwang [4].

We further observe that $\alpha=-\phi^{\prime}(1)=d / 2$.

The random trees in these three examples have also been studied by [15, [18] and [19, where the properties of a randomly selected branch are investigated. This problem is quite different, and there is no phase transition. See also [17.

Example 7.4 (Random splitting of simplices). Consider $d$-dimensional simplices, where an simplex is split into $d+1$ new simplices by choosing a random point $X$ in the interior and connecting it to the vertices of the original simplex; each new simplex has as vertices $X$ and $d$ of the original $d+1$ vertices. 
It is easily seen that this is equivalent to $d+1$-ary splitting as in Example 7.2, see [8. Lemma 3], so we have the same results as there, with $m=d+1$. In particular, $N(x)$ is asymptotically normal if $d \leq 25$.

Example 7.5 (Non-uniform splitting of intervals). Returning to binary splitting of intervals, we can generalize Example $\mathbf{7 . 1}$ by taking another distribution for the cut points; we thus have $b=2$ and $\mathbf{V}=(V, 1-V)$, where $V$ has any distribution on $(0,1)$. An interesting case is when $V$ has a beta distribution $V \sim B\left(a, a^{\prime}\right)$ with $a, a^{\prime}>0$; then

$\mathbb{E} V^{z}=B\left(a, a^{\prime}\right)^{-1} \int_{0}^{1} x^{z+a-1}(1-x)^{a^{\prime}-1} d x=\frac{B\left(a+z, a^{\prime}\right)}{B\left(a, a^{\prime}\right)}=\frac{\Gamma(z+a)}{\Gamma\left(z+a+a^{\prime}\right)} \frac{\Gamma\left(a+a^{\prime}\right)}{\Gamma(a)} ;$ $\mathbb{E}(1-V)^{z}$ is obtained by interchanging $a$ and $a^{\prime}$. In particular, if $a$ and $a^{\prime}$ are integers, then $\phi$ is rational.

We consider two special cases.

(i) The symmetric case with $a^{\prime}=a, V \sim B(a, a)$. Then

$$
\phi(z)=2 \frac{\Gamma(z+a)}{\Gamma(z+2 a)} \frac{\Gamma(2 a)}{\Gamma(a)}=\frac{\Gamma(z+a)}{\Gamma(z+2 a)} \frac{\Gamma(1+2 a)}{\Gamma(1+a)} .
$$

We have $\alpha=-\phi^{\prime}(1)=H_{2 a}-H_{a}$, with $H_{x}$ as in Example [7.2, Numerical solution of the characteristic equation seems to show that $\operatorname{Re} \lambda_{2}<1 / 2$ if and only if $a<a_{0}$, where $a_{0} \approx 59.6$.

(ii) The case $a^{\prime}=1, V \sim B(a, 1)$. Then

$\phi(z)=\frac{\Gamma(z+a)}{\Gamma(z+a+1)} \frac{\Gamma(a+1)}{\Gamma(a)}+\frac{\Gamma(z+1)}{\Gamma(z+a+1)} \Gamma(a+1)=\frac{a}{z+a}+\frac{\Gamma(z+1)}{\Gamma(z+a+1)} \Gamma(a+1)$.

One finds $\alpha=H_{a} /(a+1)$. The characteristic equation $\phi(\lambda)=1$ is equivalent to $\Gamma(a+1) \Gamma(\lambda+1) / \Gamma(\lambda+a+1)=\lambda /(\lambda+a)$ or

$$
\frac{\Gamma(a+\lambda)}{\Gamma(\lambda)}=\Gamma(a+1) .
$$

When $a=m$ is an integer, this is the same as (7.1), so $\operatorname{Re} \lambda_{2}<1 / 2$ for integer $a$ if and only if $a \leq 26$. In general, numerical solution of the characteristic equation seems to show that $\operatorname{Re} \lambda_{2}<1 / 2$ if and only if $a<a_{0}$, where $a_{0} \approx 26.9$.

\section{Non-EXAMPLES}

In this section, we give a few examples where our theorems are not valid.

Example 8.1 (Lattice). In the lattice case, there exists $R>1$ such that every $V_{j} \in\left\{R^{-k}: k \geq 1\right\} \cup\{0\}$ a.s. In this case, $\phi$ is periodic with period $2 \pi \mathrm{i} / \ln R$; in particular, the characteristic equation (1.5) has infinitely many roots $1+2 \pi \mathrm{in} / \ln R$ on $\{\lambda: \operatorname{Re} \lambda=1\}$, and thus Condition $\mathrm{B}(\delta)$ fails. Indeed, it is obvious from (1.2) that $N(x)=N\left(R^{m}\right)$ when $R^{m} \leq x<R^{m+1}$, so $\mathbb{E} N(x) / x$ oscillates and does not 
converge as $x \rightarrow \infty$. The natural approach is to consider only $x \in\left\{R^{m}: m \geq 0\right\}$. It is then straightforward to prove an analogue of Theorem 3.1, using the lattice versions of the renewal theory theorems that were used in Section 3. An analogue of Theorem 1.3 then follows by the usual (discrete) contraction method, as in 24. We leave the details to the reader.

Example 8.2 (Deterministic). If $V=\left(V_{1}, \ldots, V_{b}\right)$ is deterministic, then so is $N(x)$, and it is meaningless to ask for an asymptotic distribution. However, it makes sense to study the asymptotics of $N(x)=m(x)$. (Clearly, $\sigma^{2}(x)=0$.)

If $V$ is non-lattice, then $N(x) / x \rightarrow \alpha$ by Theorem 3.1 and Remark 3.2. If $V$ is lattice, we consider, as in Example 8.1, only $x=R^{m}, m \geq 1$.

We may assume that $V_{j}>0$ for each $j$. By Dirichlet's theorem [14, Theorem 201], for every $\varepsilon>0$, there exist arbitrarily large $t$ such that $\left|V_{j}^{\text {it }}-1\right|<\varepsilon$ for $j=1, \ldots, b$; thus $\lim \sup _{t \rightarrow \infty}|\phi(1+\mathrm{i} t)|=1$. Hence Condition $\mathrm{B}(1)$ does not hold, and therefore, by Lemma 2.1. Condition $\mathrm{B}(\delta)$ does not hold for any $\delta \leq 1$.

More precisely, if $\left|V_{j}^{\mathrm{it}}-1\right|<\varepsilon$ for $j=1, \ldots, b$, let $z_{0}=1+\mathrm{it}$. Then $\left|\phi\left(z_{0}\right)-1\right|<\varepsilon$ and

$$
\left|\phi^{\prime}\left(z_{0}\right)+\alpha\right|=\left|\sum_{j=1}^{b} \ln V_{j}\left(V_{j}^{1+i t}-V_{j}\right)\right| \leq \varepsilon \alpha .
$$

Since further $\left|\phi^{\prime \prime}(z)\right| \leq \sum_{j}\left|\ln V_{j}\right|^{2}$ for $\operatorname{Re} z \geq 0$, it follows easily that if $\varepsilon$ is small enough, then $\phi(z)-1$ has a zero in the $\operatorname{disc} B:=\left\{z:\left|z-z_{0}\right|<2 \varepsilon / \alpha\right\}$. (Use the Newton-Raphson method, or Rouché's theorem and a comparison with the linear function $\phi\left(z_{0}\right)+\left(z-z_{0}\right) \phi^{\prime}\left(z_{0}\right)$.) It follows that there exists a sequence $\lambda_{n} \in \Lambda$ with $\operatorname{Re} \lambda_{n} \rightarrow 1$ and $\operatorname{Im} \lambda_{n} \rightarrow+\infty$.

We give some concrete examples:

$V=(1 / 2,1 / 2)$ is lattice with $R=2$ and $N\left(2^{n}\right)=2^{n}$.

$V=\left(\tau^{-1}, \tau^{-2}\right)$ where $\tau=(1+\sqrt{5}) / 2$ (the golden ratio) is lattice with $R=\tau$ and $N\left(\tau^{n}\right)=F_{n+3}-1, n \geq 0$, as is easily proven by induction. ( $F_{n}$ denotes the Fibonacci numbers.) Thus, $N\left(\tau^{n}\right) \sim 5^{-1 / 2} \tau^{n+3}$.

$V=(1 / 3,2 / 3)$ is non-lattice and thus $N(x) \sim \alpha^{-1} x$, where $\alpha=\frac{1}{3} \ln 3+$ $\frac{2}{3} \ln (3 / 2)=\ln 3-\frac{2}{3} \ln 2$.

\section{Some RELATED MODELS}

The basic model may be varied in various ways. We mention here some variations that we find interesting. We do not consider these versions in the present paper; we leave the possibility of extensions of our results as an open problem, hoping that these remarks will be an inspiration for future research.

Remark 9.1. By our assumptions, the label of a node equals the sum of the labels of its children. Another version would be to allow a (possibly random) loss at each 
node. One important case is Rényi's parking problem [26, where a node with label $x$ is interpreted as an interval of length $x$ on a street, where cars of length 1 park at random. Each car splits an interval of length $x \geq 1$ into two free intervals with the lengths $U(x-1)$ and $(1-U)(x-1)$, where $U \sim \mathrm{U}(0,1)$. An obvious generalization is to split $(x-1)$ using an arbitrary random vector $\left(V_{1}, \ldots, V_{b}\right)$. (The one-sided version, where we study only one branch of the tree, is studied in [15], 17].)

Remark 9.2. Krapivsky, Ben-Naim and Grosse [21, 20] have studied a fragmentation process where fragmentation stops stochastically, with a probability $p(x)$ of further fragmentation that in general depends on the size $x$ of the fragment. Our process is the case $p(x)=\mathbf{1}_{\{x \geq 1\}}$. Another interesting case is $p(x)=1-e^{-x}$, see Remark 9.3 below.

Remark 9.3. Our model is a continuous version of the split trees studied by Devroye [8], where the labels are integers (interpreted as numbers of balls to be distributed in the corresponding subtree) and each label $n$ is, except at the leaves, randomly split according to a certain procedure into $b$ integers summing to $n-s_{0}$; here $s_{0}$ is a small positive integer (for example 1) that represents the number of balls stored at the node. Typical examples are binary search trees, $m$-ary search trees and quadtrees. We can regard the continuous model as an approximation of the discrete, or conversely, and it is easy to guess that many properties will have similar asymptotics for the two models. This has been observed in several examples by various authors, see [7] and [4. For example, the results for Example 7.2 parallel those found for $m$-ary search trees by [22, [5], [12, [3] and others. Similarly, the results in Example 7.3 parallel those found for quadtrees by 4 .

We study only the continuous version in this paper. It would be very interesting to be able to rigorously transfer results from the continuous to the discrete version (or conversely); we will, however, not attempt this here.

Note that for binary search trees, we have $n$ random (uniformly distributed) points in an interval, split the interval by the first of these points, and continue recursively splitting each subinterval that contains at least one of the points. If we scale the initial interval to have length $n$, then the probability that a subinterval of length $x$ contains at least one point is $\approx 1-e^{-x}$. Thus it seems likely that the binary search tree is well approximated by a fragmentation tree, with $\mathbf{V}$ as in Example 17.1, with a fragmentation probability $1-e^{-x}$ as in Remark 9.2. The same goes for random quadtrees and simplex trees corresponding to Examples 7.3 and 7.4

\section{REFERENCES}

[1] Asmussen, S. (1987). Applied Probability and Queues. John Wiley \& Sons, Chichester. 
[2] Bickel, P. J. and Freedman, D. A. (1981). Some asymptotic theory for the bootstrap. Ann. Statist. 9, 1196-1217.

[3] Chauvin, B. and Pouyanne, N. (2004). $m$-ary search trees when $m \geq 27$ : a strong asymptotics for the space requirements. Random Structures Algorithms 24, 133-154.

[4] Chern, H.-H., Fuchs, M. and Hwang, H.-K. (2006). Phase changes in random point quadtrees. ACM Trans. Algorithms, to appear.

[5] Chern, H.-H. and Hwang, H.-K. (2001). Phase changes in random $m$-ary search trees and generalized quicksort. Random Structures Algorithms 19, 316-358.

[6] Dall'Aglio, G. (1956). Sugli estremi dei momenti delle funzioni di ripartizione doppia. Ann. Scuola Norm. Sup. Pisa 10, 35-74.

[7] Dean, D. S. and Majumdar, S. N. (2002). Phase transition in a random fragmentation problem with applications to computer science. J. Phys. A: Math. Gen. 35, L501-L507.

[8] Devroye, L. (1999). Universal limit laws for depths in random trees. SIAM J. Comput. 28, 409-432.

[9] Feller, W. (1971). An Introduction to Probability Theory and its Applications, Volume II, 2nd ed., John Wiley \& Sons, New York.

[10] Fill, J. A. and Janson, S. (2000). A characterization of the set of fixed points of the Quicksort transformation. Electronic Comm. Probab. 5, no. 9, 77-84.

[11] Fill, J. A. and Kapur, N. (2004). The space requirement of $m$-ary search trees: distributional asymptotics for $m \geq 27$. Proceedings of the 7th Iranian Statistical Conference, Tehran 2004.

[12] Fill, J. A. and Kapur, N. (2005). Transfer theorems and asymptotic distributional results for $m$-ary search trees. Random Structures Algorithms 26, 359-391.

[13] Garnett, J. B. (1981). Bounded Analytic Functions, Academic Press, New York.

[14] Hardy, G. H. and Wright, E. M. (1960). An Introduction to the Theory of Numbers. 4th ed., Oxford Univ. Press, Oxford.

[15] Itoh, Y. and Mahmoud, H. (2003). One-sided variations on interval trees. J. Appl. Prob. 40, $654-670$.

[16] Jagers, P. (1975). Branching Processes with Biological Applications, John Wiley \& Sons, Chichester.

[17] Janson, S. (2004). One-sided interval trees. Journal of the Iranian Statistical Society 3, 149164.

[18] Javanian, M., Mahmoud, H. and Vahidi-Asl, M. (2004). Paths in $m$-ary interval trees. Discrete Mathematics 287, 45-53.

[19] Javanian, M. and Vahidi-Asl, M. (2004). Multidimensional interval trees. Mathematics and Computer Science III, Algorithms, Trees, Combinatorics and Probabilities (Vienna 2004), Eds. M. Drmota, P. Flajolet, D. Gardy, B. Gittenberger, Birkhäuser, Basel, 2004, pp. 255-256.

[20] Krapivsky P. L., Ben-Naim, E. and Grosse, I. (2004). Stable distributions in stochastic fragmentation. J. Phys. A: Math. Gen. 37, 2863-2880.

[21] Krapivsky P. L., Grosse, I. and Ben-Naim, E. (2000). Scale invariance and lack of self-averaging in fragmentation. Phys. Rev. E 61, R993-R996.

[22] Mahmoud, H.M., and Pittel, B. (1989). Analysis of the space of search trees under the random insertion algorithm. J. Algorithms 10, 52-75.

[23] Major, P. (1978). On the invariance principle for sums of independent identically distributed random variables. J. Multivariate Anal. 8, 487-517.

[24] Neininger, R. and Rüschendorf, L. (2004). A general limit theorem for recursive algorithms and combinatorial structures. Ann. Appl. Probab. 14, 378-418.

[25] Rachev, S. T. (1991). Probability Metrics and the Stability of Stochastic Models. John Wiley. 
[26] Rényi, A. (1958). On a one-dimensional random space-filling problem. (Hungarian.) Magyar Tud. Akad. Mat. Kutató Int. Közl. 3, 109-127. English transl. in Selected papers of Alfréd Rényi, Vol. II: 1956-1961. Ed. Pál Turán. Akadémiai Kiadó, Budapest, 1976, pp. 173-188.

[27] Rösler, U. and Rüschendorf, L. (2001). The contraction method for recursive algorithms. Algorithmica 29, 3-33.

[28] Sibuya, M. and Itoh, Y. (1987). Random sequential bisection and its associated binary tree. Ann. Inst. Statist. Math. 39, 69-84.

[29] Zolotarev, V. M. (1976). Approximation of the distributions of sums of independent random variables with values in infinite-dimensional spaces. (Russian.) Teor. Verojatnost. i Primenen. 21, no. 4, 741-758. Erratum ibid 22 (1977), no. 4, 901. English transl. Theor. Probability Appl. 21, no. 4, 721-737; 22 (1977), no. 4, 679-691 (1978).

[30] Zolotarev, V. M. (1977). Ideal metrics in the problem of approximating the distributions of sums of independent random variables. (Russian) Teor. Verojatnost. i Primenen. 22 (1977), no. 3, 449-465.

Department of Mathematics, Uppsala University, PO Box 480, SE-751 06 Uppsala, SWEDEN

E-mail address: svante.janson@math.uu.se

URL: http://www.math.uu.se/ svante/

Department of Mathematics and Computer Science, J.W. Goethe University, 60054

Frankfurt a.M., Germany

E-mail address: neiningr@math.uni-frankfurt.de

$U R L$ : http://www.math.uni-frankfurt.de/ neiningr/ 